\title{
The pathway to GTPase activation of elongation factor SelB on the ribosome
}

Niels Fischer ${ }^{1}$, Piotr Neumann ${ }^{2}$, Lars V. Bock ${ }^{3}$, Cristina Maracci ${ }^{4}$, Zhe Wang ${ }^{5}$, Alena Paleskava ${ }^{4} \dagger$, Andrey L. Konevega $^{4} \dagger$, Gunnar F Schröder ${ }^{5,6}$, Helmut Grubmüller ${ }^{3}$, Ralf Ficner ${ }^{2}$, Marina V. Rodnina ${ }^{4}$ \& Holger Stark ${ }^{1}$

In all domains of life, selenocysteine (Sec) is delivered to the ribosome by selenocysteine-specific tRNA (tRNA $\left.{ }^{\text {Sec }}\right)$ with the help of a specialized translation factor, SelB in bacteria. Sec-tRNA ${ }^{\mathrm{Sec}}$ recodes a UGA stop codon next to a downstream mRNA stem-loop. Here we present the structures of six intermediates on the pathway of UGA recoding in Escherichia coli by single-particle cryo-electron microscopy. The structures explain the specificity of Sec-tRNA ${ }^{\text {Sec }}$ binding by SelB and show large-scale rearrangements of Sec-tRNA ${ }^{\text {Sec }}$. Upon initial binding of SelB-Sec-tRNA ${ }^{\text {Sec }}$ to the ribosome and codon reading, the $30 \mathrm{~S}$ subunit adopts an open conformation with Sec-tRNA ${ }^{\text {Sec }}$ covering the sarcin-ricin loop (SRL) on the $50 \mathrm{~S}$ subunit. Subsequent codon recognition results in a local closure of the decoding site, which moves Sec-tRNA ${ }^{\text {Sec }}$ away from the SRL and triggers a global closure of the $30 \mathrm{~S}$ subunit shoulder domain. As a consequence, SelB docks on the SRL, activating the GTPase of SelB. These results reveal how codon recognition triggers GTPase activation in translational GTPases.

Protein synthesis on the ribosome is promoted by several GTPases. These include the universal elongation factor EF-Tu (in bacteria, or eEF1A in eukaryotes) and a specialized factor SelB ${ }^{1}$ (eEFSec-SBP2 in eukaryotes $^{2}$ ). EF-Tu and SelB deliver aminoacyl-tRNA (aa-tRNA) to the ribosome at the cost of GTP hydrolysis ${ }^{3,4}$. In contrast to EF-Tu, which is a carrier for all canonical aa-tRNAs, SelB is specialized to bind only the tRNA that is specific for $\mathrm{Sec}^{5,6}$. Sec-tRNA ${ }^{\mathrm{Sec}}$ recodes a UGA stop codon upstream of a structured mRNA stem-loop, the Sec insertion sequence (SECIS) ${ }^{7}$. EF-Tu and SelB share evolutionary conserved domains $1-3$, of which domain 1 binds $\mathrm{GTP}^{5,8-11}$. SelB has an additional domain 4 (winged helix motifs 1-4) that recruits the factor to the SECIS ${ }^{10}$. Sec has unique chemical properties, which make it an essential residue in the active site of key redox enzymes in all domains of life ${ }^{2}$. Defects in Sec biosynthesis and selenoprotein malfunction have been linked to an increasing number of diseases in humans ${ }^{12}$. Sec incorporation into non-selenoproteins, as well as the exploitation of the Sec machinery to insert designer amino acids, has received growing interest for biotechnological applications ${ }^{13,14}$. However, the structural basis for UGA recoding by SelB-Sec-tRNA ${ }^{\mathrm{Sec}}$ has not been elucidated yet. Here we use single-particle cryo-electron microscopy (cryo-EM) and extensive sorting of cryo-EM images to obtain snapshots of intermediates along the pathway of Sec-tRNA ${ }^{\mathrm{Sec}}$ delivery to the ribosome. The cryo-EM structures, in combination with ensemble rapid kinetics and all-atom explicit-solvent molecular dynamics simulations, provide detailed insights into the mechanism of UGA recoding by Sec and suggest a universal mechanism for GTPase activation on the ribosome.

\section{Visualizing intermediates of UGA recoding}

We reconstituted recoding complexes in vitro with $\mathrm{fMet}_{\mathrm{H}} \mathrm{tRNA} \mathrm{fMet}^{\mathrm{fin}}$ the peptidyl site and SelB-Sec-tRNA ${ }^{\mathrm{Sec}}$ stalled by the guanosine triphosphate analogue GDPNP at the aminoacyl site of the ribosome. As a model mRNA directing Sec incorporation, we used a construct with a AUG start codon and a UGA stop codon, followed by the SECIS of the formate dehydrogenase $\mathrm{H}(f d h F)$ gene $\mathrm{e}^{10,15}$. We recorded $\sim 1,000,000$ cryo-EM particle images and applied extensive hierarchical computational classification to sort the particle images into structurally homogenous sub-groups (Extended Data Fig. 1 and Methods). We obtained six distinct ribosome structures at 3.4-5.3 Å resolution, which depict the key intermediates of UGA recoding (Fig. 1a, Extended Data Fig. 2, Extended Data Table 1) and used the $3.4 \AA$ map to build de novo atomic models for the SelB-GDPNP-Sec-tRNA ${ }^{\text {Sec }}$ ternary complex on the ribosome (Fig. 1b-e).

The initial complex depicts the ribosome complex before SelBGDPNP-Sec-tRNA ${ }^{\text {Sec }}$ binding, with the SECIS flexibly bound at the mRNA entry channel, the UGA stop codon in the aminoacyl site and fMet-tRNA ${ }^{\text {fMet }}$ bound to the peptidyl site (Fig. 1a). We also obtained complexes in the transient states (reconstructed from only $\sim 1 \%$ of images, Fig. 1f) of the codon-independent initial binding of SelBGDPNP-Sec-tRNA ${ }^{\text {Sec }}$ to the SECIS and of codon reading with tRNA ${ }^{\text {Sec }}$ approaching the UGA codon. Although the overall structure of the two complexes is rather similar, with an overall root-mean-square deviation (r.m.s.d.) of 1.2 ̊̊ (Extended Data Fig. 1d), there are substantial local differences in functionally important regions, for example, an up to $\sim 14 \AA$ shift in the tRNA ${ }^{\text {Sec }}$ position. The highest resolution is provided by the $3.4 \AA$ cryo-EM map of SelB-GPDNP-Sec-tRNA ${ }^{\text {Sec }}$ stalled after codon recognition in the GTPase-activated state (Fig. 1a-d). We also obtained reconstructions of two states after SelB dissociation and Sec-tRNA ${ }^{\text {Sec }}$ accommodation in the aminoacyl site, in which the tRNAs are present in either classical or hybrid states ${ }^{16}$.

\section{Recognition of Sec-tRNA ${ }^{\mathrm{Sec}}$}

SelB binds Sec-tRNA ${ }^{\mathrm{Sec}}$ with extraordinary high affinity and discriminates against Ser-tRNA ${ }^{\text {Sec }}$, which is an on-pathway intermediate of SectRNA $^{\text {Sec }}$ biosynthesis ${ }^{4,17}$, and all other aa-tRNAs. Conversely, EF-Tu, which binds all elongator aa-tRNAs with uniform affinity, has very low affinity for Sec-tRNA ${ }^{\mathrm{Sec}}$ (ref. 17). Comparison of the present SelB-

${ }^{1}$ Department of Structural Dynamics, Max Planck Institute for Biophysical Chemistry, Am Fassberg 11, 37077 Göttingen, Germany. ${ }^{2}$ Department of Molecular Structural Biology, Institute for Microbiology and Genetics, GZMB, Georg-August University Göttingen, Justus-von Liebig Weg 11, 37077 Göttingen, Germany. ${ }^{3}$ Department of Theoretical and Computational Biophysics, Max Planck Institute for Biophysical Chemistry, Am Fassberg 11, 37077 Göttingen, Germany. ${ }^{4}$ Department of Physical Biochemistry, Max Planck Institute for Biophysical Chemistry, Am Fassberg 11, 37077 Göttingen, Germany. ${ }^{5}$ Institute of Complex Systems (ICS-6), Forschungszentrum Jülich, 52425 Jülich, Germany. ${ }^{6}$ Physics Department, Heinrich-Heine Universität Düsseldorf, 40225 Düsseldorf, Germany. †Present addresses: Molecular and Radiation Biophysics Department, B.P. Konstantinov Petersburg Nuclear Physics Institute of National Research Centre 'Kurchatov Institute', 188300 Gatchina, Russia (A.P., A.L.K.); St Petersburg Polytechnic University, Polytechnicheskaya, 29, 195251 St Petersburg, Russia (A.P., A.L.K.). 

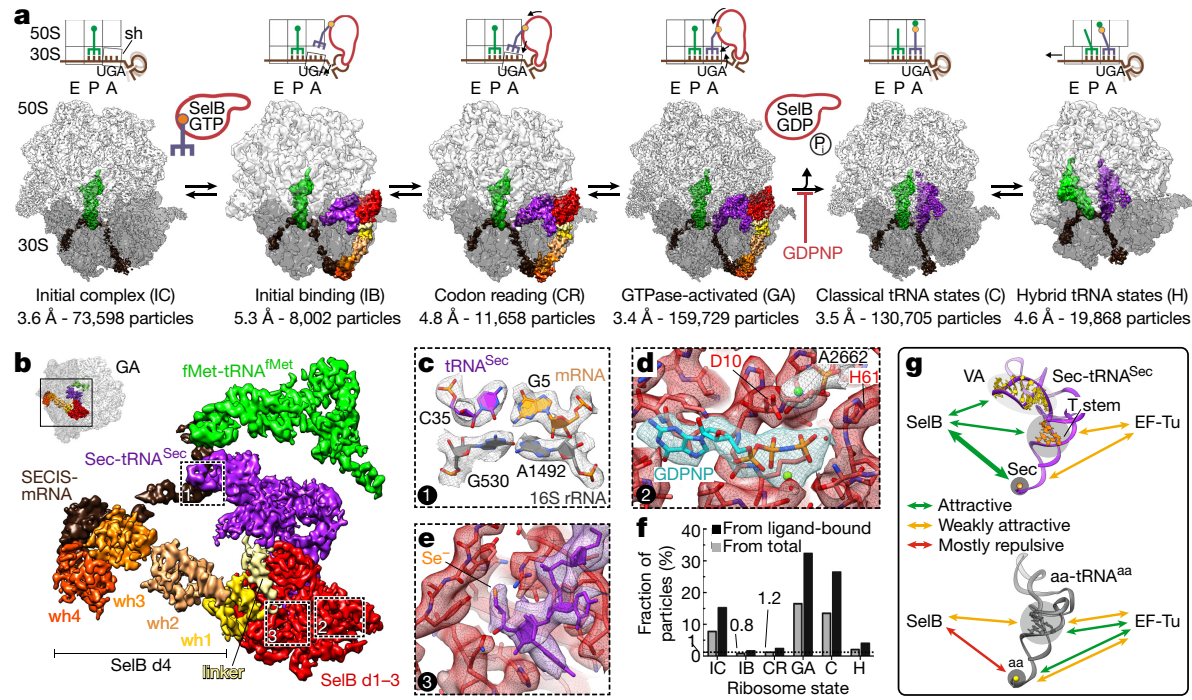

Figure 1 | Structures of intermediates along the pathway of UGA recoding by SelB-Sec-tRNA ${ }^{\mathrm{Sec}}$. a, Overview of the structures. Top row: Schematics of complexes with tRNA binding sites of the ribosome (A, P, E), UGA stop codon; sh, 30S subunit shoulder. Bottom row, cryo-EM reconstructions of distinct intermediates with the resolution (in $\AA$ ) and the number of particles for each state. IC, initial complex; IB, initial binding; CR, codon reading; GA, GTPase-activated; C, classical tRNA; and $\mathrm{H}$, hybrid tRNA states.b, Structure of SelB-GDPNP-Sec-tRNA ${ }^{\mathrm{Sec}}$ and the

GDPNP-Sec-tRNA ${ }^{\text {Sec }}$ structures with the known crystal structure of EF-Tu-GTP-Cys-tRNA ${ }^{\text {Cys }}$ (ref. 11) explains the specificity of tRNA ${ }^{\text {Sec }}$ recognition (Fig. 1g, Extended Data Fig. 3). The linker (Leu340Gln363; Escherichia coli numbering used throughout) between domains 3 and 4 of SelB, which is absent in EF-Tu, binds and distorts the extralong variable arm of tRNA ${ }^{\mathrm{Sec}}$ (Extended Data Fig. 3a-c, l). Furthermore, an extended loop in SelB domain 3 (Ile326-Asn330) facilitates interactions with the acceptor- and T-stems of tRNA ${ }^{\mathrm{Sec}}$ at the antideterminant box that distinguishes tRNA ${ }^{\text {Sec }}$ from canonical tRNAs ${ }^{18}$. The corresponding loop in EF-Tu adopts a different conformation that allows sequence-specific interactions with canonical tRNAs ${ }^{19,20}$, but impedes interactions with tRNA ${ }^{\text {Sec }}$ (Extended Data Fig. 3e, f, j). Finally, the amino-acid-binding pocket of SelB is lined with positively charged residues (Extended Data Fig. 3g-i), allowing SelB to specifically recognize the negatively charged selenol group and to discriminate against Ser-tRNA ${ }^{\text {Sec }}$. In EF-Tu the amino-acid-binding pocket is negatively charged; this, together with the low affinity of EF-Tu for the tRNA ${ }^{\text {Sec }}$ body ${ }^{21}$, explains why EF-Tu does not bind Sec-tRNA ${ }^{\text {Sec }}$. Notably, the variable arm of tRNA ${ }^{\text {Sec }}$ undergoes a large-scale rotation of about $50^{\circ}$ upon release of Sec-tRNA ${ }^{\text {Sec }}$ from SelB and accommodation in the aminoacyl site (Extended Data Fig. 31). This rotation is essential to allow binding of the next aa-tRNA to the aminoacyl site after peptidyltRNA ${ }^{\text {Sec }}$ translocation to the peptidyl site.

\section{SelB-specific domain 4}

The SECIS recruits domain 4 of SelB ${ }^{10}$ and may guide the ternary complex towards the ribosome. We constructed a model for an early recruitment complex by docking SelB-Sec-tRNA ${ }^{\mathrm{Sec}}$ onto the SECIS of the initial complex (Fig. 2a). In the model, SelB and Sec-tRNA ${ }^{\text {Sec }}$ do not interact with the ribosome, but a rotation would move SelB-Sec-tRNA ${ }^{\text {Sec }}$ into its position in the initial binding state. SECIS-dependent tethering via domain 4 is SelB-specific, as EF-Tu is recruited by the L7/12 stalk of the $50 \mathrm{~S}$ ribosomal subunit ${ }^{22}$. In the subsequent states domain 4 serves as a flexible anchor for SelBSec-tRNA ${ }^{\text {Sec }}$ (Fig. 2b, Extended Data Fig. 4a, b). When SelB moves towards the GTPase-activated state, domain 4 maintains its interactions with the SECIS and the adjacent 30 S elements (helix h16 of 16 S ribosomal RNA (rRNA) and protein S4) and follows mainly the movements of the
SECIS on the ribosome in the GTPase-activated state at $3.4 \AA$ resolution. d, domains of SelB; wh, winged helix motifs. Numbers and dashed boxes indicate the regions depicted in c-e. c, Recognition of the UGA codon by the anticodon of tRNA ${ }^{\mathrm{Sec}}$. d, The GTPase centre of SelB in the activated state. e, Recognition of the selenol group $\left(\mathrm{Se}^{-}\right)$by SelB. $\mathbf{f}$, Fraction of particles in each intermediate relative to the total number of particles $(\sim 1,000,000)$ or to the number of particles with ligand density $(\sim 500,000)$. g, Scheme of tRNA recognition by SelB versus EF-Tu. VA, variable arm.

30S subunit. These rigid-body motions of domain 4 do not affect the remaining part of SelB, because the highly flexible linker connecting domains 3 and 4 uncouples the two parts.

\section{Requirements for SelB GTPase activation}

GTP hydrolysis by EF-Tu is a major checkpoint that controls the rate and fidelity of decoding ${ }^{23}$. Rapid GTP hydrolysis by EF-Tu is triggered by the correct codon-anticodon interaction ${ }^{24}$; the requirements for the GTPase activation in SelB are unknown. In the presence of the correct SECIS and the UGA codon, the rate of GTP hydrolysis in SelB- $\left[\gamma_{-}^{32} \mathrm{P}\right]$ GTP-Sec-tRNA ${ }^{\text {Sec }}$ on the ribosome is about $3 \mathrm{~s}^{-1}$, which is four orders of magnitude higher than the intrinsic GTPase activity of the ternary complex in the absence of ribosomes (Fig. 2c, d, Extended Data Fig. 4c-f). Mutation of three key recognition bases in the SECIS ${ }^{10,25}$ results in a 600-fold reduction of the GTPase rate, in line with the role of the SECIS as a tether for SelB (see above and refs 10,26). With a correct SECIS element, but a non-cognate UUC codon in the aminoacyl site, the rate of GTP hydrolysis is also reduced by 600 -fold compared to the cognate complex (Fig. 2d), suggesting that recognition of the cognate UGA codon by Sec-tRNA ${ }^{\text {Sec }}$ is essential for GTPase activation. Thus, the requirement for the correct codon-anticodon interaction as a trigger for GTP hydrolysis is similar for SelB and EF-Tu. For EF-Tu, the existence of several pre-hydrolysis steps, including initial binding and codon reading, has been demonstrated by ensemble kinetics and single molecule FRET experiments ${ }^{27,28}$. The similarities between SelB and EF-Tu as to the reaction pathway and codon-dependence of the GTPase activation suggest a conserved mechanism of decoding and aa-tRNA selection, with GTP hydrolysis being a crucial step between initial selection and proofreading $24,29,30$.

\section{Structural dynamics of the 30 S subunit}

Pioneering crystallographic studies on complexes of isolated 30S subunits demonstrated that cognate codon-anticodon complex formation triggers local rearrangements of three universally conserved bases of $16 \mathrm{~S}$ rRNA in the decoding centre (G530, A1492, and A1493) and a global domain closure in the $30 \mathrm{~S}$ subunit ${ }^{31,32}$. Here we describe the rearrangements of the 30S subunit upon UGA codon recognition by Sec-tRNA ${ }^{\mathrm{Sec}}$ in the context of the $70 \mathrm{~S}$ ribosome (Fig. 3, Extended Data Fig. 5) 

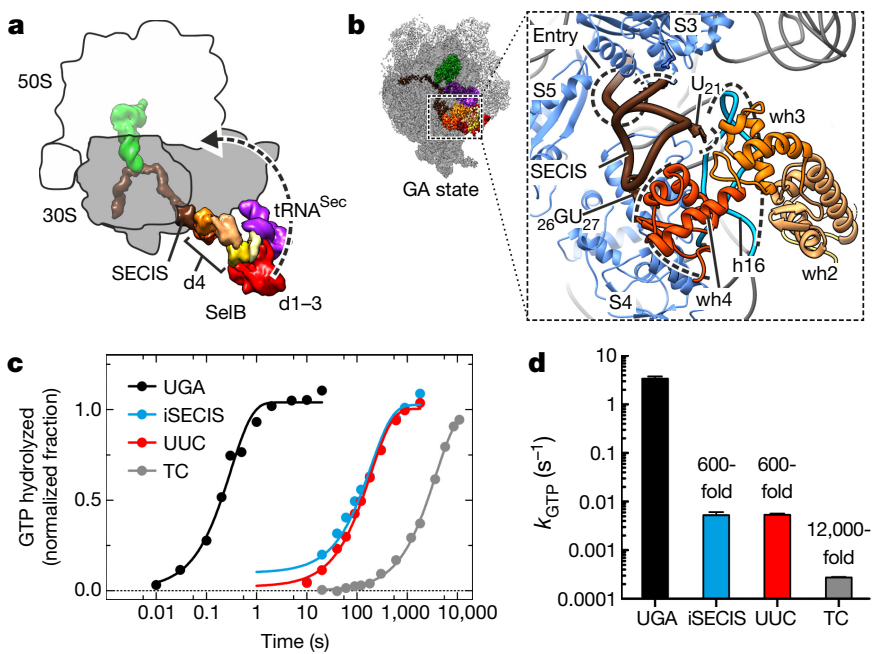

Figure $2 \mid$ Roles of SelB domain 4 and the GTPase activation on the ribosome. a, A model for the ribosome-independent recruitment of SelB onto the SECIS guiding SelB-Sec-tRNA ${ }^{\mathrm{Sec}}$ to its position in the initial binding state (arrow). b. Interactions of SelB and the SECIS at the mRNA entry site (entry) in the GTPase-activated state marked by dashed contours; the interactions are maintained in the initial binding and codon reading states (data not shown). h16, helix of $16 \mathrm{~S}$ rRNA; S3-S5, proteins S3 to S5. c, Time courses of GTP hydrolysis by SelB on ribosomes programmed with mRNA comprising a correct SECIS and a cognate UGA codon (UGA), or an incorrect SECIS and a cognate UGA codon (iSECIS), or a correct SECIS, but a non-cognate codon (UUC), and by the free SelBGTP-Sec-tRNA ${ }^{\text {Sec }}$ (ternary complex; TC). d, The reduction in the GTP hydrolysis rates as compared to complexes carrying the cognate codon and correct SECIS (UGA); error bars, s.e.m. of the exponential fitting with $n=2$ for UGA and $n=3$ for iSECIS, UUC and ternary complex.

and show how these changes contribute to the mechanism of GTPase activation of SelB (Fig. 4).

In the initial complex before SelB-GDPNP-Sec-tRNA ${ }^{\text {Sec }}$ binding, the $30 \mathrm{~S}$ subunit is in a non-rotated classical state ${ }^{33,34}$ (Fig. 3a, Extended Data Fig. 5d). The A1492 and A1493 bases appear to be in dynamic equilibrium between 'flipped-in' and 'flipped-out' states with respect to $\mathrm{h} 44$ of $16 \mathrm{~S}$ rRNA. G530 is in the anti-conformation which is maintained in all subsequent states. Initial binding of SelB-GDPNP-Sec-tRNA ${ }^{\mathrm{Sec}}$ is codon-independent, as evident from the large distance between $\mathrm{tRNA}^{\mathrm{Sec}}$ anticodon and UGA codon (10 A, Fig. 3a, Extended Data Fig. 5b). In the initial binding state, the flipped-in conformation of the A1492 and A1493 bases is stabilized and the aminoacyl site is open (Fig. 3a). The $30 \mathrm{~S}$ shoulder rotates outwards, away from the $50 \mathrm{~S}$ subunit. This $30 \mathrm{~S}$ domain opening widens the intersubunit space, which may be important to facilitate initial binding of SelB domain 2 to the 30 S shoulder or to prevent premature docking of SelB on the 50S subunit (see below). Further progression of tRNA ${ }^{\mathrm{Sec}}$ towards the mRNA codon in the codon reading state may allow the interaction between the third codon base and the anticodon of tRNA ${ }^{\mathrm{Sec}}$, but this state is still incompatible with full codon-anticodon complex formation (Fig. 3a, Extended Data Fig. 5b). In the GTPase-activated state, the codon is recognized, the codonanticodon helix adopts the Watson-Crick geometry, and A1492 and A1493 are stabilized in the flipped-out position, which results in a local closure of the aminoacyl site. Together with G530, the adenines interact with the minor groove of the codon-anticodon duplex at the first and second codon position. At the same time, the $30 \mathrm{~S}$ subunit undergoes a large-scale domain closure, which mainly involves an inward rotation of the $30 \mathrm{~S}$ shoulder and decreases the distance spanned by the SelBGDPNP-Sec-tRNA ${ }^{\mathrm{Sec}}$ complex. During all these steps, the universally conserved A1913 in helix 69 (H69) of 23S rRNA interacts with A1492 and A1493 in h44 in a state-specific fashion and acts to stabilize the flipped-in position of the two adenines in the initial binding and codon reading states (Extended Data Fig. 5a). The closed conformation of
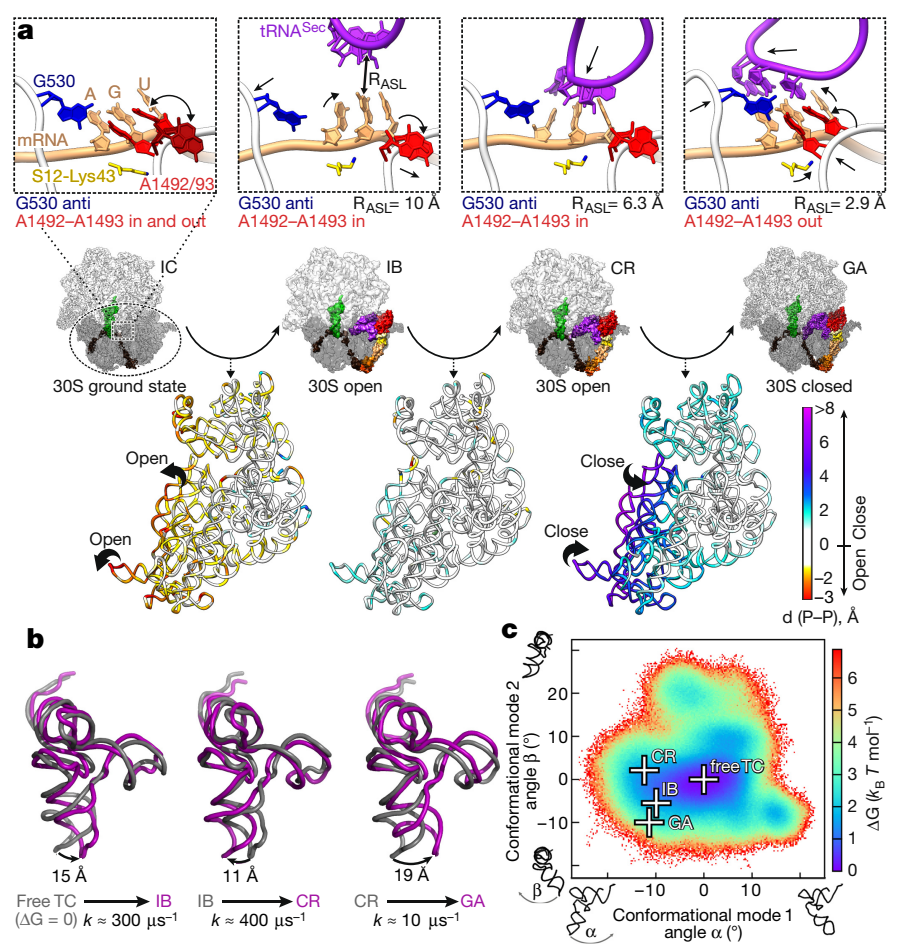

Figure 3 | Structural changes of $30 \mathrm{~S}$ subunit and tRNA during UGA decoding. a, Coupling between local and global movements of the $30 \mathrm{~S}$ subunit. Top row, local changes in the decoding centre. Middle row, overview of the states. Bottom row, global rearrangements of the $30 \mathrm{~S}$ subunit. The heat map quantifies the displacement of $16 \mathrm{~S}$ rRNA backbone phosphate atoms between consecutive states as obtained by superposition on $23 \mathrm{~S}$ rRNA of the initial complex; negative values designate domain opening, positive values domain closure. $\mathrm{R}_{\mathrm{ASL}}$, distance between $\mathrm{RNA}^{\mathrm{Sec}}$ anticodon (N3 of C35) and UGA (N1 of G). b. Conformations of Sec-tRNA ${ }^{\mathrm{Sec}}$ Rearrangements from the free ternary complex SelB-GTP-Sec-tRNA ${ }^{\text {Sec }}$ to the initial binding state (left), from initial binding to codon reading (middle) and codon reading to GTPase-activated states (right). The deviations of the anticodon tip are measured in $\AA$ at the phosphate of C35. The free ternary complex structure and interconversion rates $(k)$ were obtained by molecular dynamics simulation from c. c, Free-energy landscape of tRNA ${ }^{\mathrm{Sec}}$ conformations in the free ternary complex obtained by $24-\mu$ s all-atom explicit-solvent molecular dynamics simulations (Methods). Crosses denote the tRNA conformations seen in the respective states on the ribosome; the free-energy minimum corresponds to the major conformer of the free ternary complex. The heat map denotes the free energy.

the 30 S subunit in the GTPase-activated state is practically identical to that observed for the canonical EF-Tu-GDPCP-Trp-tRNA ${ }^{\text {Trp }}$ ternary complex on the ribosome $\mathrm{e}^{35}$. However, compared to the states preceding codon recognition, the rearrangement is more extensive in the present complexes than in those inferred from the structures of isolated $30 \mathrm{~S}$ subunit complexes ${ }^{31}$ (Fig. 3a).

\section{Conformational changes of tRNA ${ }^{\mathrm{Sec}}$}

The aa-tRNA has an essential role in signalling rearrangements at the decoding site to the 50S subunit, leading to GTPase activation of EF-Tu ${ }^{36-38}$. Our cryo-EM data show marked structural changes in $\mathrm{tRNA}^{\mathrm{Sec}}$ on the ribosome, which affect three main areas of the tRNA ${ }^{\mathrm{Sec}}$ molecule: the variable arm, the elbow region consisting of the $\mathrm{D}$ - and T-loops, and the D-stem-anticodon-stem region (Fig. 3b). The D-and T-loops move closer to each other in the initial binding state and change only slightly in other states. By contrast, the anticodon- and D-stems undergo large-scale distortions in each transition. In the GTPaseactivated state $\mathrm{tRNA}^{\mathrm{Sec}}$ adopts a conformation similar to that of the canonical aa-tRNAs in the EF-Tu-ribosome complex ${ }^{35,38}$. In principle, the observed large-scale conformational rearrangements of the tRNA could present a kinetic barrier that controls the GTPase activation in 

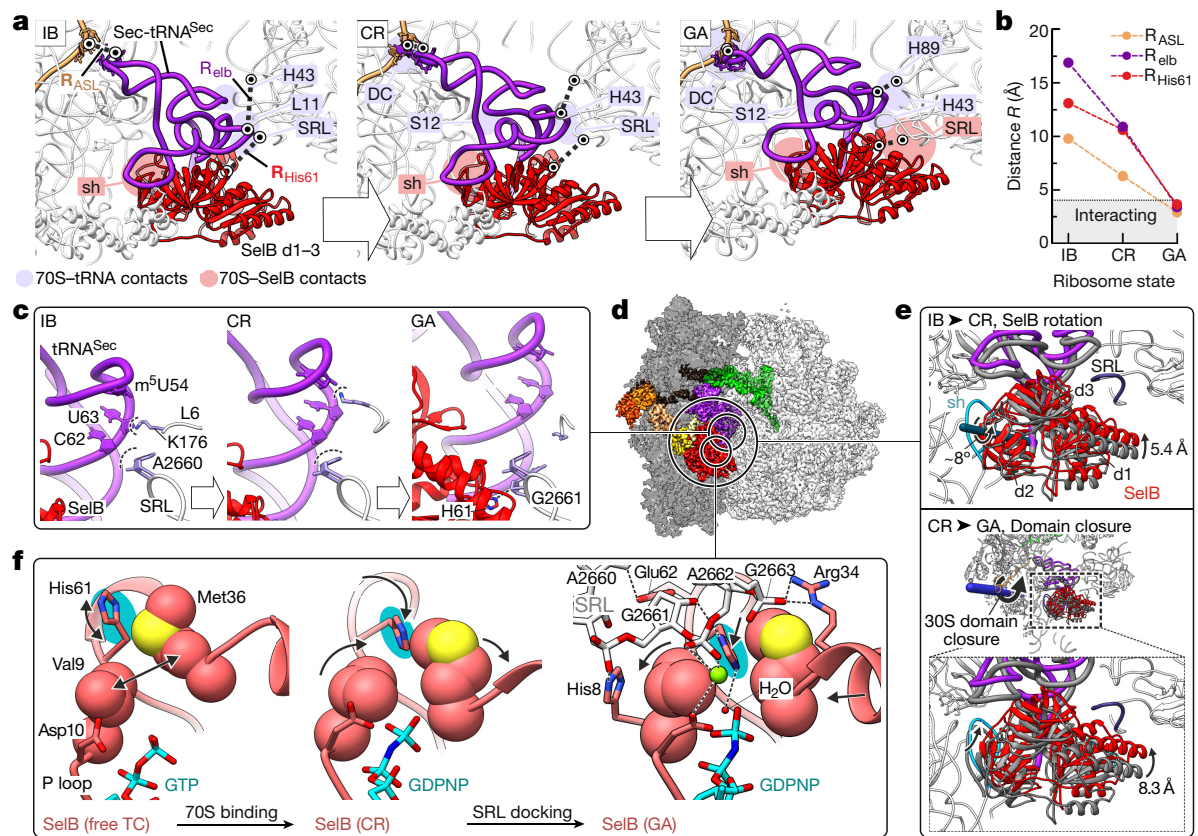

Figure 4 | Sequential docking of SelB-Sec-tRNA ${ }^{\text {Sec }}$ on the SRL.

a, Movements of the tRNA ${ }^{\mathrm{Sec}}$ anticodon $\left(\mathrm{R}_{\mathrm{ASL}}\right)$ and elbow $\left(\mathrm{R}_{\mathrm{elb}}\right)$ and of SelB His61 ( $\left.\mathrm{R}_{\mathrm{His} 61}\right)$; sh, 30S shoulder (G357 to U368 of $16 \mathrm{~S}$ rRNA); DC, decoding centre; $\mathrm{H} 43$ and H89, helices of $23 \mathrm{~S}$ rRNA. b. State-specific distance changes. $R_{\mathrm{ASL}}$ (N3 of C35 in tRNA ${ }^{\mathrm{Sec}}$ to N1 of G in UGA); $\mathrm{R}_{\mathrm{elb}}$ (tRNA ${ }^{\mathrm{Sec}}$ elbow, $\mathrm{C5}^{\prime}$ of $\Psi 55$ in tRNA ${ }^{\mathrm{Sec}}$ to O2' of A2473 in H89); $\mathrm{R}_{\mathrm{His} 61}$ (ND1 of His61 in SelB to O2' of G2661 in SRL). c, SRL interactions with Sec-tRNA ${ }^{\text {Sec }}$ and SelB His61. d, Overview of the ribosome-SelB complex in the GTPase-activated state indicating the areas for close-ups in $\mathbf{c}, \mathbf{e}, \mathbf{f}$. e, Docking of SelB onto the SRL. Distances are measured at C $\alpha$ of Ala120.
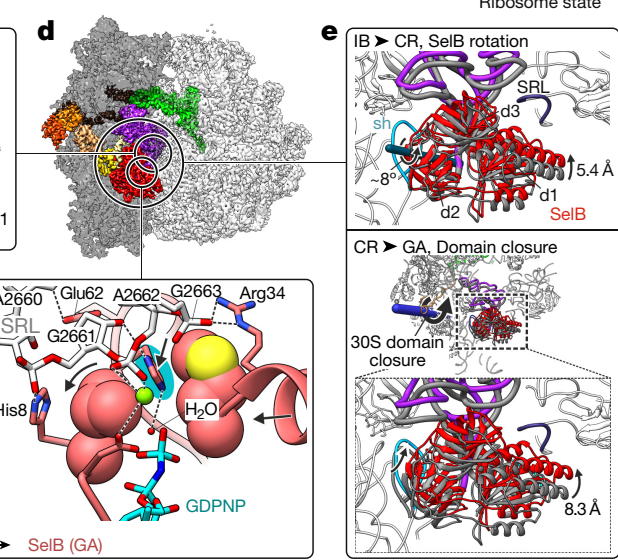

Top, rotation of SelB relative to the $30 \mathrm{~S}$ shoulder (sh) upon transition from initial binding to codon reading states. Bottom, rotation of SelB together with tRNA ${ }^{\mathrm{Sec}}$ and the $30 \mathrm{~S}$ shoulder upon $30 \mathrm{~S}$ domain closure in the GTPase-activated state. f, The GTPase site. Left, free ternary complex. The two-headed arrows denote spontaneous fluctuations of SelB His61 (cyan) and of Val9 and Met36. Middle, inactive flipped-in conformation of His61 upon ribosome binding in the codon reading state, potentially stabilized by Val9. Right, active conformation of SelB docked on the SRL in the GTPase-activated state stabilized by the ribosome-SelB interaction network. the complex. To understand the dynamics of those rearrangements, we carried out $24-\mu$ s all-atom explicit-solvent molecular dynamics simulations of SelB-GTP-Sec-tRNA ${ }^{\text {Sec }}$ (Fig. 3c, Extended Data Fig. 6a, b; Methods). We mapped the free-energy conformational landscape and identified the lowest-energy structural model of the ternary complex free in solution. All tRNA ${ }^{\text {Sec }}$ conformations observed on the ribosome are sampled spontaneously within less than a microsecond in solution (Fig. 3c). Given that the GTPase activation takes milliseconds (Fig. 2c), the conformational changes of the tRNA, though clearly essential, do not seem to be rate-limiting for the GTPase activation. Of the large pool of potential tRNA ${ }^{\mathrm{Sec}}$ structures accessible in solution, the ribosome selectively stabilizes specific subsets of conformations depending on the sum of all interactions in a given state (Fig. 3b, c), thereby rectifying the structural fluctuations of the complexes towards the GTPase-activated state.

\section{Docking of SelB onto the SRL}

The SRL constitutes the key activator of the GTPase activity for all translational GTPases ${ }^{39}$. Notably, docking of the SelB GTP-binding domain on the SRL occurs only upon formation of the GTPase-activated state (Fig. 4a). Whereas the tRNA ${ }^{\mathrm{Sec}}$ anticodon moves towards the codon in the $30 \mathrm{~S}$ decoding centre, the elbow region changes its interactions from the initial contact with the SRL and protein L11 (initial binding state) to SRL and $\mathrm{H} 43$ of $23 \mathrm{~S}$ rRNA (codon reading state) before docking onto H89 of $23 \mathrm{~S}$ rRNA (Fig. 4a). The movements of the tRNA ${ }^{\mathrm{Sec}}$ anticodon towards the codon and of the tRNA elbow towards H89, as well as the formation of the GTPase-activated state (monitored by the position of the key GTPase residue His61 in SelB) are coupled (Fig. 4b). These motions control the progression of SelB towards the GTPase-activated state, in line with the major role of tRNA in signalling codon recognition ${ }^{36-38}$.

Stepwise tRNA ${ }^{\mathrm{Sec}}$ docking is facilitated by several elements of the ribosome, including the L11-rRNA arm (consisting of protein L11 and helices H43 and H44 of 23S rRNA), the SRL (together with protein L6), protein $\mathrm{S} 12$, and $\mathrm{H} 69$ of $23 \mathrm{~S}$ rRNA that guide $\mathrm{RNA}^{\mathrm{Sec}}$ to the decoding centre. The L11-rRNA arm acts as a relay that hands over the tRNA during transitions from state to state (Extended Data Fig. 7a). Binding of tRNA ${ }^{\mathrm{Sec}}$ results in a $>10 \AA$ displacement of the entire L11-rRNA in the initial binding state. In subsequent states, the L11-rRNA arm relaxes stepwise into its initial position. Notably, in the initial binding and codon reading states, the SRL interacts with tRNA ${ }^{\mathrm{Sec}}$, rather than with SelB (Fig. 4c). A2660 acts as a platform for docking of the T-stem residues of tRNA ${ }^{\mathrm{Sec}}$, but the contacts with $\mathrm{tRNA}^{\mathrm{Sec}}$ change between the initial binding and codon reading states. Further movement of tRNA ${ }^{\mathrm{Sec}}$ in the GTPase-activated state disrupts these contacts and enables docking of SelB onto the SRL. On the 30S subunit, protein S12 engages the acceptor stem of $\mathrm{tRNA}^{\mathrm{Sec}}$ in the codon reading state and serves as a pivot for tRNA ${ }^{\text {Sec }}$ rotation into the GTPase-activated state (Extended Data Fig. 7b). A1913 of H69, in turn, reaches into the decoding centre and may help to guide the anticodon towards the codon in the aminoacyl site during the transition from the codon reading to the GTPaseactivated state by interacting with the functionally important modified residue isopentenyl-A37 in tRNA ${ }^{\text {Sec }}$ (ref. 40; Extended Data Fig. 5a).

In the initial binding, codon reading and GTPase-activated states, SelB maintains its interactions with $\mathrm{tRNA}^{\mathrm{Sec}}$ and follows its movements (Fig. 4a, e). SelB domain 2 remains bound to the shoulder of $16 \mathrm{~S}$ rRNA, while adapting to the changes in the position of $\mathrm{tRNA}^{\mathrm{Sec}}$. Upon transition from the initial binding to the codon reading state, this adaptation requires a rotation of domain 2 relative to the $30 \mathrm{~S}$ subunit shoulder, which changes the interactions and moves SelB slightly towards the 50S subunit (Fig. 4e, upper panel, and Extended Data Fig. 7c). In the GTPase-activated state, as a consequence of codon recognition and the global $30 \mathrm{~S}$ domain closure, SelB rotates together with tRNA ${ }^{\mathrm{Sec}}$ and the $30 \mathrm{~S}$ shoulder. Domain 1 of SelB-comprising several charged residues (Arg34, Glu62 and Arg116) - moves into close proximity to 


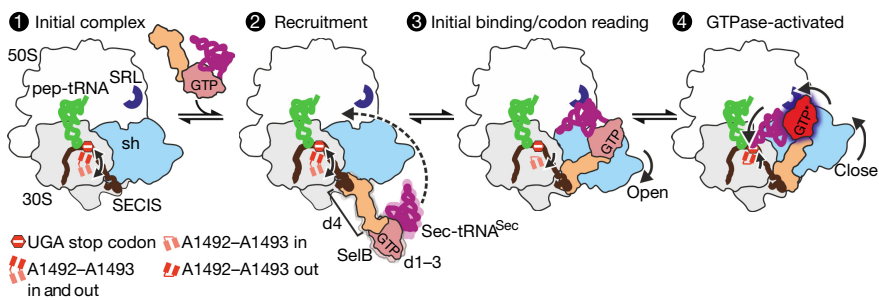

Figure 5 Mechanism of ribosome-dependent GTPase activation of SelB-Sec-tRNA ${ }^{\mathrm{Sec}}$ upon UGA recoding by Sec. See text for explanation. pep-tRNA, peptidyl-tRNA; d, domains of SelB; dark red glow, GTPase activation of SelB; arrows 'open' and 'close', domain opening and closure of the $30 \mathrm{~S}$ shoulder (sh).

the SRL (Fig. 4e, bottom panel). These residues may help to promote the small interdomain motion of SelB that is required to completely dock onto the SRL (Extended Data Fig. 7d). The molecular dynamics simulations of the free SelB-GTP-Sec-tRNA ${ }^{\text {Sec }}$ complex indicate that this interdomain motion in SelB is rapidly sampled in solution (Extended Data Fig. 7e) and largely independent of tRNA dynamics (Extended Data Fig. 6c).

\section{GTPase activation}

The active site of all translational GTPases is highly conserved. The invariant histidine (His61 in SelB, His84 in EF-Tu) is essential for rapid GTP hydrolysis in all translational GTPases ${ }^{41-43}$. The molecular dynamics simulations of SelB-GTP-Sec-tRNA ${ }^{\text {Sec }}$ in solution suggest that His61 can rapidly fluctuate between a flipped-out (pointing away from the $\gamma$-phosphate) and a flipped-in (reaching towards the $\gamma$-phosphate) conformation (Fig. 4f, Extended Data Fig. 7f, g), similarly to His 84 in $\mathrm{EF}-\mathrm{Tu}^{44,45}$. In the codon reading state the conformational equilibrium of His61 is shifted towards a partially flipped-in conformation (Fig. 4f). The bulky residue Val9 of SelB appears to stabilize this inactive conformation, thereby impeding further movement of His61 towards the nucleotide (Fig. 4f), which explains the low rate of spontaneous GTP hydrolysis before codon recognition (Fig. 2c).

In the GTPase-activated state, SelB adopts a conformation that is similar to that of the pre-hydrolysis state of EF-Tu ${ }^{35,38}$ (Fig. 4f, Extended Data Fig. 7h). The universally conserved residue G2661 of the SRL stabilizes His61 of SelB in the flipped-in conformation, pointing towards the water molecule aligned for the attack on the $\gamma$-phosphate. Val9 stacks onto the His61 imidazole ring, providing additional stabilization of the active conformer. The phosphate of A2662 coordinates a $\mathrm{Mg}^{2+}$ ion, which may be important in positioning Asp10 in SelB (homologous to Asp21 in EF-Tu, which is crucial for GTP hydrolysis ${ }^{42}$ ); a similar $\mathrm{Mg}^{2+}$ coordination is seen with the corresponding residue Asp22 of elongation factor $\mathrm{G}$ in the pre-hydrolysis state on the ribosome $\mathrm{e}^{46}$. Further SelB residues (His8, Arg34, Glu62, Arg116) stabilize the interactions with the SRL (Extended Data Fig. 7d, right panel). Given the evolutionary conservation of the residues constituting the GTPase centre, GTP hydrolysis is likely to follow the same universal pathway for all translational GTPases.

\section{Conclusions}

The present data show how SelB delivers Sec-tRNA ${ }^{\text {Sec }}$ to the ribosome, provide insights into the local and global conformations of the ribosome upon codon recognition by Sec-tRNA ${ }^{\mathrm{Sec}}$ and suggest the mechanism for GTPase activation of SelB (Fig. 5). Sec-tRNA ${ }^{\mathrm{Sec}}$ and SelB in solution can sample many different conformations. The interactions with the ribosome rectify these spontaneous fluctuations and guide the progression towards GTPase activation. In the initial complex, the SECIS element in the mRNA is exposed for SelB binding and the universally conserved bases A1492 and A1493 in the decoding centre fluctuate between flipped-in and flipped-out conformations (Fig. 5, step 1). Recruitment to SECIS tethers SelB to the vicinity of the ribosome; this contact between SelB domain 4 and the SECIS is maintained in all subsequent steps (Fig. 5, step 2). Initial binding of SelB-Sec-tRNA ${ }^{\text {Sec }}$ to the ribosome induces an open conformation of the $30 \mathrm{~S}$ subunit and stabilizes A1492 and A1493 in a flipped-in conformation, thereby facilitating codon reading (Fig. 5, step 3). Subsequent codon recognition triggers local closure of the decoding site, with A1492 and A1493 flipped out, and a global domain closure of the $30 \mathrm{~S}$ subunit. The closed conformation facilitates re-positioning of the tRNA and docking of SelB on the SRL. Docking at the SRL aligns key residues at the nucleotide binding pocket of SelB and results in GTPase activation (Fig. 5, step 4). The structure of the GTPase-activated state is likely to be universal for translational GTPases in all domains of life. The docking of GTPases onto the SRL as a result of correct codon-anticodon complex formation and $30 \mathrm{~S}$ domain closure may represent a common mechanism by which the ribosome ensures the pre-hydrolysis selection of the cognate aa-tRNA delivered by SelB, EF-Tu, or their eukaryotic homologues. This work emphasizes the power of the combination of high-resolution cryo-EM, rapid kinetics and molecular dynamics simulations for the reconstruction of multistep reaction pathways and understanding the function of dynamic molecular machines.

Online Content Methods, along with any additional Extended Data display items and Source Data, are available in the online version of the paper; references unique to these sections appear only in the online paper.

Received 11 July; accepted 24 October 2016.

Published online 14 November 2016.

1. Böck, A. Biosynthesis of selenoproteins-an overview. Biofactors 11, 77-78 (2000).

2. Lescure, A., Fagegaltier, D., Carbon, P. \& Krol, A. Protein factors mediating selenoprotein synthesis. Curr. Protein Pept. Sci. 3, 143-151 (2002)

3. Berchtold, H. et al. Crystal structure of active elongation factor Tu reveals major domain rearrangements. Nature 365, 126-132 (1993).

4. Paleskava, A. Konevega, A. L. \& Rodnina, M. V. Thermodynamic and kinetic framework of selenocysteyl-tRNASec recognition by elongation factor SelB. J. Biol. Chem. 285, 3014-3020 (2010).

5. Forchhammer, K., Boesmiller, K. \& Böck, A. The function of selenocysteine synthase and SELB in the synthesis and incorporation of selenocysteine. Biochimie 73, 1481-1486 (1991).

6. Itoh, Y., Sekine, S., Suetsugu, S. \& Yokoyama, S. Tertiary structure of bacterial selenocysteine tRNA. Nucleic Acids Res. 41, 6729-6738 (2013).

7. Zinoni, F., Heider, J. \& Böck, A. Features of the formate dehydrogenase mRNA necessary for decoding of the UGA codon as selenocysteine. Proc. Natl Acad. Sci. USA 87, 4660-4664 (1990).

8. Itoh, Y., Sekine, S. \& Yokoyama, S. Crystal structure of the full-length bacterial selenocysteine-specific elongation factor SelB. Nucleic Acids Res. 43 9028-9038 (2015).

9. Leibundgut, M., Frick, C., Thanbichler, M., Böck, A. \& Ban, N. Selenocysteine tRNA-specific elongation factor SelB is a structural chimaera of elongation and initiation factors. EMBO J. 24, 11-22 (2005)

10. Kromayer, M., Wilting, R., Tormay, P. \& Böck, A. Domain structure of the prokaryotic selenocysteine-specific elongation factor SelB. J. Mol. Biol. 262, 413-420 (1996).

11. Nissen, P., Thirup, S., Kjeldgaard, M. \& Nyborg, J. The crystal structure of Cys-tRNA ${ }^{\text {Cys }}$-EF-Tu-GDPNP reveals general and specific features in the ternary complex and in tRNA. Structure 7, 143-156 (1999).

12. Hatfield, D. L., Carlson, B. A., Xu, X. M., Mix, H. \& Gladyshev, V. N. Selenocysteine incorporation machinery and the role of selenoproteins in development and health. Prog. Nucleic Acid Res. Mol. Biol. 81, 97-142 (2006).

13. Metanis, N. \& Hilvert, D. Natural and synthetic selenoproteins. Curr. Opin. Chem. Biol. 22, 27-34 (2014)

14. Bulteau, A. L. \& Chavatte, L. Update on selenoprotein biosynthesis. Antioxid. Redox Signal. 23, 775-794 (2015).

15. Ringquist, S. et al. Recognition of the mRNA selenocysteine insertion sequence by the specialized translational elongation factor SELB. Genes Dev. 8, 376-385 (1994).

16. Moazed, D. \& Noller, H. F. Interaction of tRNA with 23S rRNA in the ribosomal A, P, and E sites. Cell 57, 585-597 (1989).

17. Förster, C., Ott, G., Forchhammer, K. \& Sprinzl, M. Interaction of a selenocysteine-incorporating tRNA with elongation factor Tu from E.coli. Nucleic Acids Res. 18, 487-491 (1990).

18. Rudinger, J., Hillenbrandt, R., Sprinzl, M. \& Giegé, R. Antideterminants present in minihelix(Sec) hinder its recognition by prokaryotic elongation factor Tu. EMBO J. 15, 650-657 (1996)

19. Yikilmaz, E., Chapman, S. J., Schrader, J. M. \& Uhlenbeck, O. C. The interface between Escherichia coli elongation factor Tu and aminoacyl-tRNA. Biochemistry 53, 5710-5720 (2014). 
20. Schrader, J. M., Chapman, S. J. \& Uhlenbeck, O. C. Understanding the sequence specificity of tRNA binding to elongation factor Tu using tRNA mutagenesis. J. Mol. Biol. 386, 1255-1264 (2009).

21. Asahara, H. \& Uhlenbeck, O. C. The tRNA specificity of Thermus thermophilus EF-Tu. Proc. Natl Acad. Sci. USA 99, 3499-3504 (2002).

22. Diaconu, M. et al. Structural basis for the function of the ribosomal $L 7 / 12$ stalk in factor binding and GTPase activation. Cell 121, 991-1004 (2005)

23. Maracci, C. \& Rodnina, M. V. Review: Translational GTPases. Biopolymers 105 , 463-475 (2016).

24. Pape, T., Wintermeyer, W. \& Rodnina, M. Induced fit in initial selection and proofreading of aminoacyl-tRNA on the ribosome. EMBO J. 18, 3800-3807 (1999).

25. Heider, J., Baron, C. \& Böck, A. Coding from a distance: dissection of the mRNA determinants required for the incorporation of selenocysteine into protein. EMBO J. 11, 3759-3766 (1992).

26. Kotini, S. B., Peske, F. \& Rodnina, M. V. Partitioning between recoding and termination at a stop codon-selenocysteine insertion sequence. Nucleic Acids Res. 43, 6426-6438 (2015)

27. Wohlgemuth, I., Pohl, C., Mittelstaet, J., Konevega, A. L. \& Rodnina, M. V. Evolutionary optimization of speed and accuracy of decoding on the ribosome. Phil. Trans. R. Soc. Lond. B 366, 2979-2986 (2011).

28. Marshall, R. A., Aitken, C. E., Dorywalska, M. \& Puglisi, J. D. Translation at the single-molecule level. Annu. Rev. Biochem. 77, 177-203 (2008).

29. Thompson, R. C., Dix, D. B. \& Karim, A. M. The reaction of ribosomes with elongation factor Tu.GTP complexes. Aminoacyl-tRNA-independent reactions in the elongation cycle determine the accuracy of protein synthesis. J. Biol. Chem. 261, 4868-4874 (1986).

30. Ruusala, T., Ehrenberg, M. \& Kurland, C. G. Is there proofreading during polypeptide synthesis? EMBO J. 1, 741-745 (1982).

31. Ogle, J. M., Murphy, F. V., Tarry, M. J. \& Ramakrishnan, V. Selection of tRNA by the ribosome requires a transition from an open to a closed form. Cell $\mathbf{1 1 1}$ 721-732 (2002).

32. Ogle, J. M. et al. Recognition of cognate transfer RNA by the $30 \mathrm{~S}$ ribosomal subunit. Science 292, 897-902 (2001).

33. Dunkle, J. A. et al. Structures of the bacterial ribosome in classical and hybrid states of tRNA binding. Science 332, 981-984 (2011)

34. Fischer, N., Konevega, A. L., Wintermeyer, W., Rodnina, M. V. \& Stark, H. Ribosome dynamics and tRNA movement by time-resolved electron cryomicroscopy. Nature 466, 329-333 (2010)

35. Voorhees, R. M., Schmeing, T. M., Kelley, A. C. \& Ramakrishnan, V. The mechanism for activation of GTP hydrolysis on the ribosome. Science $\mathbf{3 3 0}$, 835-838 (2010).

36. Piepenburg, O. et al. Intact aminoacyl-tRNA is required to trigger GTP hydrolysis by elongation factor Tu on the ribosome. Biochemistry 39 , 1734-1738 (2000)

37. Cochella, L. \& Green, R. An active role for tRNA in decoding beyond codon:anticodon pairing. Science 308, 1178-1180 (2005).
38. Schmeing, T. M., Voorhees, R. M., Kelley, A. C. \& Ramakrishnan, V. How mutations in tRNA distant from the anticodon affect the fidelity of decoding. Nat. Struct. Mol. Biol. 18, 432-436 (2011)

39. Moazed, D., Robertson, J. M. \& Noller, H. F. Interaction of elongation factors EF-G and EF-Tu with a conserved loop in 23 S RNA. Nature 334, 362-364 (1988).

40. Warner, G. J. et al. Inhibition of selenoprotein synthesis by selenocysteine tRNA[Ser]Sec lacking isopentenyladenosine. J. Biol. Chem. 275, 28110-28119 (2000).

41. Daviter, T., Wieden, H.J. \& Rodnina, M. V. Essential role of histidine 84 in elongation factor Tu for the chemical step of GTP hydrolysis on the ribosome. J. Mol. Biol. 332, 689-699 (2003)

42. Maracci, C. Peske, F. Dannies, E.,Pohl, C. \& Rodnina, M. V. Ribosome-induced tuning of GTP hydrolysis by a translational GTPase. Proc. Natl Acad. Sci. USA 111, 14418-14423 (2014).

43. Cunha, C. E. et al. Dual use of GTP hydrolysis by elongation factor $\mathrm{G}$ on the ribosome. Translation (Austin) 1, e24315 (2013)

44. Adamczyk, A. J. \& Warshel, A. Converting structural information into an allosteric-energy-based picture for elongation factor Tu activation by the ribosome. Proc. Natl Acad. Sci. USA 108, 9827-9832 (2011).

45. Wallin, G., Kamerlin, S. C. \& Aqvist, J. Energetics of activation of GTP hydrolysis on the ribosome. Nat. Commun. 4, 1733 (2013).

46. Tourigny, D. S., Fernández, I. S., Kelley, A. C. \& Ramakrishnan, V. Elongation factor $\mathrm{G}$ bound to the ribosome in an intermediate state of translocation. Science 340, 1235490 (2013).

Acknowledgements We thank F. Würriehausen, A. Bursy, O. Geintzer, S. Kappler C. Kothe, T. Niese, T. Wiles, and M. Zimmermann for expert technical assistance and M. Lüttich and T. Koske for support in high-performance computation. The work was supported by the Deutsche Forschungsgemeinschaft Grant FOR 1805 (to H.S., M.V.R. and H.G.) and by the Sonderforschungsbereich 860 (to R.F.).

Author Contributions N.F. conceived the project, performed cryo-EM experiments and data analysis and drafted the paper. P.N. built atomic models and performed pseudo-crystallographic refinement. L.V.B. performed and analyzed molecular dynamics simulations. C.M. performed the kinetic analysis. A.P. and A.L.K prepared ribosome complexes for cryo-EM. Z.W. and G.F.S performed homology modelling. N.F., M.V.R. and H.S. finalized the paper with inputs from all authors.

Author Information Reprints and permissions information is available at www.nature.com/reprints. The authors declare no competing financial interests. Readers are welcome to comment on the online version of the paper. Correspondence and requests for materials should be addressed to N.F. (niels.fischer@mpibpc.mpg.de) and H.S. (hstark1@gwdg.de).

Reviewer Information Nature thanks A. Amunts, M. Wahl and S. Yokoyama for their contribution to the peer review of this work. 


\section{METHODS}

Ribosomes, factors and RNAs. Ribosomes from E. coli MRE 600, initiation factor (IF1, IF2, IF3), and fMet-tRNA ${ }^{\mathrm{fMet}}$ were prepared as described ${ }^{47-49}$. E. coli SelA and SelD (gift from M. Wahl, Free University of Berlin) and SelB ${ }^{50}$ containing a hexahistidine tag (gift from A. Böck, LMU Munich) were expressed in BL21(DE3) cells and purified according to published protocols ${ }^{4,51}$. Biochemical analysis showed that SelB carrying the His-tag is fully functional in the interactions with guanine nucleotides, SECIS elements, and Sec-tRNA ${ }^{\mathrm{Sec}}$ and in mediating UGA recoding in vivo ${ }^{4,50}$. RNA $^{\mathrm{Sec}}$ was overexpressed in E. coli BL21 transformed with the plasmid pCB2013 (gift from A. Böck, LMU Munich) ${ }^{52}$ and purified and aminoacylated as described ${ }^{4,53,54}$. The mRNA UGA was a derivative of $m L P 75 s^{54}$ (Extended Data Fig. 4d). The templates for mRNAs UUC and iSECIS (Extended Data Fig. 4e, f) were prepared by PCR mutagenesis. Transcription and refolding of the mRNAs were performed as described ${ }^{5,55}$.

Complex preparation for cryo-EM. $70 \mathrm{~S}$ ribosomes $(3 \mu \mathrm{M})$ were incubated with IF1, IF2, IF3 $(4.5 \mu \mathrm{M})$, mRNA $(15 \mu \mathrm{M})$, and f $\left[{ }^{3} \mathrm{H}\right] \mathrm{Met}^{\mathrm{M}}$-tRNA ${ }^{\mathrm{fMet}}(7 \mu \mathrm{M})$ in buffer A (50 mM Tris- $\mathrm{HCl}$ (pH 7.5), $70 \mathrm{mM} \mathrm{NH}_{4} \mathrm{Cl}, 30 \mathrm{mM} \mathrm{KCl}, 7 \mathrm{mM} \mathrm{MgCl}_{2}, 2 \mathrm{mM}$ DTT) with $1 \mathrm{mM} \mathrm{GTP} \mathrm{for} 30 \mathrm{~min}$ at $37^{\circ} \mathrm{C}$. Initiation efficiency was close to $100 \%$ as verified by nitrocellulose binding. Initiation complexes were purified by gel filtration on a Biosuite $450 \mathrm{HR} 5 \mu \mathrm{m}$ column (Waters). To prepare the ribosomeSelB complexes, ternary complexes SelB-GDPNP-Sec-tRNA ${ }^{\text {Sec }}$ were prepared in buffer B (50 mM Hepes-KOH, pH 7.5, $70 \mathrm{mM} \mathrm{NH}_{4} \mathrm{Cl}, 30 \mathrm{mM} \mathrm{KCl}, 7 \mathrm{mM} \mathrm{MgCl}_{2}$, $2 \mathrm{mM}$ DTT) by incubating SelB $(1 \mu \mathrm{M})$ with GDPNP $(2 \mathrm{mM})$ for $4 \mathrm{~min}$ at $37^{\circ} \mathrm{C}$ adding Sec-tRNA ${ }^{\text {Sec }}(1 \mu \mathrm{M})$ and incubating for $2 \mathrm{~min}$ at $23^{\circ} \mathrm{C}$. Ternary complex $(0.5 \mu \mathrm{M})$ was incubated with initiation complex $(0.06 \mu \mathrm{M})$ at $0^{\circ} \mathrm{C}$ in buffer $\mathrm{B}$ supplemented with $0.6 \mathrm{mM}$ spermine and $0.4 \mathrm{mM}$ spermidine before application onto EM grids.

GTPase assay. Initiation complexes were prepared as described ${ }^{42}$. Ternary complex SelB- $\left[\gamma^{32} \mathrm{P}\right]$ GTP-Sec-tRNA ${ }^{\mathrm{Sec}}$ was prepared in buffer A, by incubating SelB $(9 \mu \mathrm{M})$ with $\left[\gamma^{32} \mathrm{P}\right] \mathrm{GTP}(60 \mu \mathrm{M})$ for $20 \mathrm{~min}$ at room temperature (RT), followed by addition of Sec-tRNA ${ }^{\mathrm{Sec}}(9 \mu \mathrm{M})$ and incubation for $2 \mathrm{~min}$ at room temperature. Unbound $\left[\gamma^{32} \mathrm{P}\right] \mathrm{GTP}$ was removed by gel filtration ${ }^{56}$. The GTPase activity of SelB was determined at single round conditions, by mixing purified ternary complex $(0.05 \mu \mathrm{M})$ with UGA, iSECIS and UUC mRNA-programmed initiation complexes at the indicated concentrations. Intrinsic GTP hydrolysis was measured in the absence of ribosomes. When necessary, quench-flow experiments were performed in a KinTek apparatus. Reactions were quenched with formic acid $(25 \% \mathrm{v} / \mathrm{v})$ and the extent of GTP cleavage was determined by thin layer chromatography and phosphor imaging ${ }^{57}$. The rate of GTP hydrolysis was determined by exponential fitting of the time courses using GraphPad Prism software (GraphPad Software, Inc.); time courses were normalized to the respective reaction end levels.

Cryo-EM analysis. Cryo-EM grids were prepared by applying $5 \mu \mathrm{l}$ of initiation complex-SelB-GDPNP-Sec-tRNA ${ }^{\text {Sec }}$ complexes onto EM grids (Quantifoil $3.5 / 1 \mu \mathrm{m}$, Jena) covered with pre-floated continuous carbon and subsequently vitrified using a Vitrobot Mark IV (FEI Company, Eindhoven) operated at $4{ }^{\circ} \mathrm{C}$ and $100 \%$ humidity. $4,000 \times 4,000$ images $(12,681$ in total) were acquired in the integration mode by spot-scanning $(3 \times 3$ images per $3.5 \mu \mathrm{m}$ hole in the Quantifoil carbon film) on a Falcon 2 direct detector (FEI Eindhoven) using a Titan Krios microscope (FEI Eindhoven), fitted with a XFEG electron source (FEI Eindhoven) and a spherical aberration (Cs)-corrector (CEOS Heidelberg). Acquisition was at $300 \mathrm{kV}$ acceleration voltage, an electron dose of $\sim 30 \pm 5$ electrons per $\AA^{2}$ (determination based on calibration with a Faraday cup), -0.7 to $-2.6 \mu \mathrm{m}$ defocus and a nominal magnification of $118,000 \times$ resulting in a final pixel size of $\sim 1.16 \AA$ (determined by optimizing correlation of the final 3D map with atomic coordinates from X-ray crystallography ${ }^{58}$ ). The Cs-corrector was tuned as described ${ }^{58}$ to reduce electron-optical aberrations, linear distortion (to $<0.1 \%$ ) and axial coma (usrimageshift tuning) resulting from the spot-scanning procedure. Ribosome particle images were selected and corrected locally for the contrast-transfer function as described ${ }^{58}$. Good quality particle images were selected according to power spectra quality (that is, showing Thon rings better than $3.5 \AA$ ), yielding a total of 969,526 ribosome particles. An initial $3 \mathrm{D}$ structure was computed from randomly selected 100,000 particles to judge the compositional and conformational homogeneity of the image dataset. Even at low-resolution $(\sim 10 \AA)$ this average structure showed only very weak, scattered density in the factor-binding region, which disappeared completely upon further refinement to higher-resolution ( 3.7 $)$ (Extended Data Fig. 1c) This observation indicated that the occupancy of ribosome particles with SelBSec-tRNA ${ }^{\text {Sec }}$ was low, in line with the low Sec incorporation efficiency in vivo and in vitro ${ }^{26}$

The dataset was sorted computationally in a hierarchical manner ${ }^{34}$ (Extended Data Fig. 1a, b). First, images were sorted according to global ribosome conformation, as described ${ }^{58}$ (step 1), which allowed us to discard low quality particles and 50S subunits. Subsequently (step 2), particles were sorted according to ligand occupancy using supervised classification by projection matching ${ }^{59}$ on the basis of a library of ribosome-ligand cryo-EM maps ${ }^{34,60}$. Importantly, this library entailed a cryo-EM map of the canonical ribosome-EF-Tu complex ${ }^{60}$ as potential reference for the ribosome-SelB complex to avoid any reference bias for SelB-Sec-tRNA ${ }^{\mathrm{Sec}}$, for which no structure was available. To further avoid any high-resolution reference bias, sorting in both steps was performed using low-pass filtered reference maps and particle images binned to about $6.9 \AA$ per pixel. The resulting maps of ribosome-ligand complexes showed all specific features expected for the Sec system (that is, the SECIS in the mRNA, the extra-long variable arm of tRNA ${ }^{\mathrm{Sec}}$, and domain 4 of SelB). In step 3, the resulting populations of ribosome-ligand particles were further rectified from low-quality particles by three-dimensional (3D) classification in Relion 1.2 and 1.3 (ref. 61). Focused 3D classification in Relion 1.3 without alignment was used to isolate three particle sub-populations that show distinct states of SelB-Sec-tRNA ${ }^{\mathrm{Sec}}$ on the ribosome (step 4); particle images were coarsened to $3.16 \AA$ per pixel for this step to improve the speed and robustness of classification. Finally, to further enrich for ribosome particles of good quality containing SelB within these sub-populations, supervised classification was used in step 5 to assign particles either to the respective ribosome-SelB complex or ribosomes with only peptidyl-site tRNA, but not SelB-Sec-tRNA ${ }^{\mathrm{Sec}}$ bound; again a pixel sampling of $3.16 \AA$ and low-pass filtered reference maps were used to avoid any high-resolution reference bias. In a similar manner, the particle populations of ribosomes carrying only the peptidyl-site tRNA or two tRNAs in the classical state were further computationally cleaned up by supervised classification. The six homogenous ribosome-ligand particle populations resulting from this sorting procedure were refined to high resolution according to the gold-standard procedure in Relion 1.3 and overall resolution of the final maps was determined using highresolution noise substitution ${ }^{62}$ in Relion 1.4 (Extended Data Fig. 2a). The final cryo-EM maps were sharpened as described ${ }^{58}$; and for each reconstruction local, resolution maps were computed using Resmap ${ }^{63}$ (Extended Data Fig. 2f, g). If not denoted otherwise, densities in the figures are rendered at $2 \sigma$, except winged-helix motifs 3-4 and SECIS of the GTPase-activated state, which are rendered at $1.3-1.7 \sigma$. Figures were prepared using UCSF Chimera ${ }^{64}$.

Atomic model refinement. Pseudo-crystallographic refinement and model building. For initial model building and refinement, the cryo-EM density map of the GTPase-activated state was sharpened by applying a B factor of $-120 \AA^{2}$, filtered to the estimated highest resolution limit and masked using a pseudo bulk solvent envelope. The mask was obtained by merging the cryo-EM map filtered at different frequencies $\left(9.0 \AA, 6.0 \AA\right.$ and $4.0 \AA$ ) using the RAVE package ${ }^{65}$ and UCSF Chimera $^{64}$. The masked cryo-EM density map was converted to reciprocal space structure factors using Crystallography and NMR System $(\mathrm{CNS})^{66,67}$ without employing phase significance blurring scale factors derived from FSC values.

Initial rigid body fit of an atomic model of the kirromycin-stalled $E$. coli ribosome complex ${ }^{58}$ lacking EF-Tu and Phe-tRNA ${ }^{\text {Phe }}$ was performed against the masked cryo-EM map of the GTPase-activated state using Chimera, followed by rigid body refinement of individual chains in PHENIX ${ }^{68}$ program. The atomic model of SelB with exception of tandem winged-helix motifs 3 and 4 was obtained by combining manual rebuilding and homology modelling with density-guided energy optimization, as implemented in the Rosetta package ${ }^{69,70}$, employing a template derived from Methanococcus maripaludis SelB (PDB ID: 4AC9) ${ }^{9}$ and alignment provided by the HHPRED server ${ }^{71}$. SelB tandem winged-helix motifs (wh) 1 and 2 were modelled based on the crystal structure of the isolated domain 4 of SelB from Moorella thermoacetica (PDB ID: 1LVA) ${ }^{72}$; and wh3 and wh4 with mRNA SECIS were modelled on the basis of the crystal structure of $E$. coli SECIS RNA bound to the domain of elongation factor SelB (PDB ID: 2PJP) ${ }^{73}$ using Rosetta and $\mathrm{Coot}^{74}$. Sec-tRNA ${ }^{\mathrm{Sec}}$ was manually modelled on the basis of the crystal structure of tRNA ${ }^{\text {Sec }}$ in complex with seryl-tRNA synthetase (PDB ID: 3 W3S) ${ }^{6}$ with Coot and Rcrane ${ }^{75}$ and optimized by ERRASER ${ }^{76}$. The catalytic water molecule in the GTPase active centre of SelB was modelled on the basis of the superposition from PDB ID $4 \mathrm{~V} 5 \mathrm{~L}^{35}$ into available density. Before global optimization of the atomic model of ribosome was performed, models of all individual protein chains were relaxed against the masked cryo-EM map filtered to $3.8 \AA$ resolution (GTPase-activated state) using Rosetta. The assembled complete ribosome model was further minimized in both real and reciprocal space (ERRASER, phenix. real_space_refine ${ }^{77}$, phenix.refine) with alternating cycles of manual rebuilding in Coot and monitoring the local fit to the density with RESOLVE ${ }^{78}$. Refinement progress was additionally monitored by calculating the Pearson correlation coefficient $\left(\mathrm{CC}_{\text {work }}\right.$; ref. 79$)$, as well as the Fourier shell correlation between the cryo-EM reconstruction and a model map $\left(\mathrm{FSC}_{\text {work }}\right)$. Both real and reciprocal space refinements in PHENIX employed automatically generated restraints and additional pseudo DEN (Deformable Elastic Network) restraints in order to maintain chemically important interactions. Group atomic displacement factors (ADP) were refined exclusively during reciprocal space refinement steps. During real space 
refinement steps in phenix.real_space_refine Ramachandran plot restraints were enabled. For parts of the model exhibiting larger conformational differences and/or lower local map resolution, additional cycles of real space refinement and manual fitting were performed against experimental map filtered to lower resolution, which was gradually increased between subsequent refinement steps until convergence. To maintain the intermolecular interactions of selected model fragments within local environment, all residues within at least $15 \AA$ radius were included. For final refinement steps the cryo-EM map was sharpened ${ }^{58}$ and masked using a smoothed model-based envelope generated within $3.0 \AA \AA$ radius around atoms of the ribosome model of the GTPase-activated state before conversion to reciprocal space structure factors; for these steps and improved visualization the cryo-EM maps resolved at $\leq 3.6 \AA$ resolution (initial complex, classical and GTPase-activated states) were resampled to a pixel size of $0.789 \AA$. The final model of the GTPase-activated state consisting of 154,136 individual atoms was refined to $24.90 \%$ and 0.936 for $R_{\text {work }}$ and $\mathrm{CC}_{\text {work }}$ (definition is given below), respectively. The model of the GTPaseactivated state exhibits a good stereochemistry with $90.87 \%$ of residues in the most favoured region and $0.88 \%$ residues in the disallowed region of the Ramachandran plot, protein side chain outliers of $2.83 \%$ and all atom clash score 15.09 .

The remaining states (initial complex, initial binding, codon reading, classical and hybrid states) were modelled based on the final model of GTPase-activated state in an analogous way. For cryo-EM reconstructions resolved at a resolution lower than $3.6 \AA$, a $3.8 \AA$ radius for generating a smooth model-based envelope was chosen before generating reciprocal space structure factors used for the final refinement steps. $\mathrm{FSC}_{\text {work }}$ and $\mathrm{CC}_{\text {work/overall, }}$ were calculated in a resolution-dependent manner using SFALL program (CCP4 suite ${ }^{80}$. Real space correlation coefficients (RSCC) were calculated using RESOLVE. All atomic models fit well the experimental cryo-EM map as judged based on the following criteria. (1) The Pearson correlation coefficient $\left(\mathrm{CC}_{\text {work }}\right)$ calculated between model and map structure factors ( $F_{\text {model }}$ and $F_{\mathrm{EM}}$ ) used for refinement is greater than 0.2 for the highest resolution shell and the overall correlation coefficient $\left(\mathrm{CC}_{\text {overall }}\right)$ is not lower than 0.9. (2) The calculated FSC $_{\text {work }}$ value between model map coefficients $\left(F_{\text {model }}\right.$, phase $\left.e_{\text {model }}\right)$ and structure factors derived from the cryo-EM map $\left(\mathrm{F}_{\mathrm{EM}}\right.$, phase $\left.\mathrm{EM}_{\mathrm{EM}}\right)$ used for refinement are not lower than 0.5 for the highest resolution shell and overall greater than 0.9. (3) The cumulative RSCC values are greater than $0.8,0.6$ and 0.4 for $53-75 \%$, $88-94 \%$ and $95-97 \%$ of the residues, respectively. Detailed refinement statistics are presented in Extended Data Table 1, FSC and CC $_{\text {work }}$ curves in Extended Data Fig. 2a-c.

Modelling of the recruitment complex. To build the initial recruitment complex, SelB domain 4 from the structure of the isolated SECIS-domain 4 complex (wh2 to wh4; PDB ID: $2 \mathrm{PLY})^{73}$ was docked onto the initial complex by superimposing the SECIS. Then, wh2 was used to model our structure of SelB-GDPNPSec-tRNA ${ }^{\text {Sec }}$ onto the initial complex-domain 4 complex.

Refinement against half maps. Refinements of final models against datasets obtained from two half maps were performed at $2.5 \AA$ resolution in PHENIX using several cycles of real space refinement followed by reciprocal space refinement employing reference model restraints. To remove possible model bias a random shift of $0.3 \AA$ was applied to all atomic positions before real space refinement. The FSC and CC were calculated between the models and the masked half-maps used for refinement, as well as between the model and the other half-map for crossvalidation. The individual half-maps were masked using a smoothed mask derived from the respective refined model using a radius of 3.0 or $3.8 \AA$ depending on the estimated highest resolution limit.

Molecular dynamics simulations. Simulation setup. To obtain the energetics and dynamics of the free ternary complex, molecular dynamics simulations were started from the ribosome-bound conformations (initial binding, codon reading, and GTPase-activated states). Coordinates of Sec-tRNA ${ }^{\mathrm{Sec}}$, SelB (amino acids 1-401), and GTP were extracted from the cryo-EM structures along with resolved water molecules and ions in the vicinity $(<5 \AA)$. The system was protonated, solvated, and ions were added as described earlier ${ }^{81}$

All simulations were carried out with Gromacs 5 (ref. 82) using the amberff12sb force field ${ }^{83}$, and the SPC/E water mode ${ }^{84}$. Parameters for potassium and chloride ions were taken from ref. 85 and for modified nucleotides from Aduri et al. ${ }^{86}$. Atom types for selenocysteine were obtained with ANTECHAMBER ${ }^{87}$ and partial charges were determined using DFT-B3LYP with a $6-31 / G^{*}$ basis set. Bond and virtual-site constraints, temperature and pressure coupling were applied as described ${ }^{81}$

For each of the three starting structures, the system was pre-equilibrated as described ${ }^{81}$ with potential-energy minimization and 50-ns molecular dynamics simulations with position restraints followed by release of position restraints during 20 ns. Production runs started at $70 \mathrm{~ns}$. At times 170, 270, and $370 \mathrm{ns,}$ coordinates were extracted from the trajectory, new velocities were assigned and new simulations were started ( 12 simulations; total production run simulation time of $24 \mu \mathrm{s})$.
Conformational dynamics of the ternary complex. To address the question of whether intrinsic conformational changes are rate-limiting for the transitions between the free ternary complex and the ribosome-bound states, we carried out a principal component analysis (PCA $)^{88}$ on three subsets of atoms: ' $\mathrm{V}$-arm' (P-atoms of variable arm), 'no-v-arm' (remaining peptidyl-atoms of tRNA), and 'D1' (CQ-atoms of SelB domain D1). The trajectories were rigid-body fitted using T-stem and acceptor-stem peptidyl-atoms for the 'v-arm' and 'no-v-arm' subsets and $\mathrm{D} 2 \mathrm{C} \alpha$-atoms for the 'D1' subsets ( $\Delta t=10 \mathrm{ps}$, discarding first $200 \mathrm{~ns}$ ). The trajectories were concatenated and the atomic-displacement covariance-matrix was calculated. The first eigenvectors, sorted according to their eigenvalues, represent the most dominant conformational modes. For 'no-v-arm', the first two eigenvectors largely consist of a bending motion of the anticodon arm. To estimate the free-energy landscape of this motion, the projections of all frames onto these eigenvectors were binned. The relative free energy of each bin was calculated via $\Delta G_{\text {bin }}=-k_{\mathrm{B}} T \ln \left(c_{\mathrm{bin}} / c_{\mathrm{max}}\right)$, where $k_{\mathrm{B}}$ is the Boltzmann constant, $T$ the temperature, $c_{\text {bin }}$ the number of frames in the bin and $c_{\max }$ is the maximum of all $c_{\text {bin }}$ (Fig. $4 \mathrm{c}$, Extended Data Figs 6a, 7e).

For 'no-v-arm' conformational modes 1 and 2 , the bending angles ( $\alpha$ and $\beta$ ) as a function of the projection onto the respective mode were calculated. The vector between the centres of mass of U34-A36 peptidyl-atoms and of T-stem peptidyl-atoms was calculated. For each projection, the angle between the vector and the vector corresponding to the bin with $\Delta G=0$ was calculated.

To monitor the local geometry of the active site in the free ternary complex, the minimal distances between His61 imidazole atoms and GTP atoms $\left(R_{\mathrm{GTP}}\right)$ as well as the van-der-Waals distance between Val9 and Met36 $\left(R_{\text {Gate }}\right)$ were calculated ( $\Delta t=10 \mathrm{ps}$, discarding first $200 \mathrm{~ns}$ ). The resulting free-energy landscape is shown in Extended Data Fig. 7f.

Transition rates for tRNA movements. To estimate the magnitudes of rates for transitions of tRNA ${ }^{\mathrm{Sec}}$ in the free ternary complex between the conformations corresponding to the ribosome-bound states, the projections onto the "no-v-arm" conformational modes 1 and 2 were calculated, thus obtaining 2-dimensional trajectories. For each simulation the number of transitions $n$ between the region around the free-energy minimum (all bins with $0 \leq \Delta G \leq 0.5 k_{\mathrm{B}} T \mathrm{~mol}^{-1}$ ) and the ribosome-bound conformations was counted. For each ribosome-bound tRNA conformation a region was defined by an ensemble of 10,000 structures generated from the cryo-EM coordinates and b-factors. For each peptidyl-atom and ensemble structure, the coordinate obtained from cryo-EM was shifted in a random direction by distance $d$ drawn from a normal distribution $p(d)$. The normal distribution $p(d)$ with $\mu=0$ and $\sigma=\operatorname{rmsf}$ (calculated from the $b$-factor) was set to zero for $d<\sigma$ and $d>\sigma$. The resulting structures were projected onto the 'no-v-arm' conformational modes. The region was defined as all bins with entries from these structures. The transition rates were calculated for each simulation by dividing $n$ by the simulation time $t_{\text {sim. }}$. The mean rates and standard deviations were obtained by weighting the individual transition rates by the corresponding $t_{\text {sim. }}$.

Coupling of tRNA and SelB conformational modes. To address a possible coupling of 'v-arm' and 'no-v-arm' conformational modes, we investigated whether the projections onto these modes are correlated as would be expected for coupled motions. For all four pairs of eigenvectors $\mathbf{v}_{\mathrm{a}}$ and $\mathbf{v}_{\mathrm{b}}$, where $\mathbf{v}_{\mathrm{a}}$ is either 'no-v-arm' mode 1 or 2 and $\mathbf{v}_{\mathrm{b}}$ is either " $\mathrm{v}$-arm" mode 1 or 2 , the correlation coefficient of the projections was calculated. To estimate the statistical error, a bootstrapping method was applied. First, the autocorrelation time $\tau$ was defined by $f_{\text {ac }}(\tau)=\exp (-1)$, where $f_{\text {ac }}(\Delta t)$ is the autocorrelation function of the projection. For a given pair of eigenvectors $\mathbf{v}_{\mathrm{a}}$ and $\mathbf{v}_{\mathrm{b}}$, the maximum of the two corresponding $\tau$ values $\left(\tau_{\max }\right)$ was used as an interval to resample the projections, resulting in sets of $N=t_{\text {sim }} / \tau_{\max }$ projections. This resampling was repeated 1,000 times and mean and standard deviations of the correlation coefficients were calculated for the simulations started from the individual ribosome-bound conformations and for all simulations combined (Extended Data Fig. 6b, red points). To check whether the resulting correlation coefficients are statistically significant, the standard deviations expected from uncorrelated projections were estimated by randomly drawing $N$ projections $(1,000$ repetitions; Extended Data Fig. 6b, grey points).

To address coupling between tRNA dynamics and motions of SelB domain 1 (D1), the same analysis was carried out for 'no-v-arm' and 'D1' conformational modes (Extended Data Fig. 6c).

Data availability statement. Cryo-EM maps/coordinates of the atomic models for each state have been deposited in the Electron Microscopy Data Bank/Protein Data Bank (http://www.emdatabank.org/) with the following accession codes: initial complex, EMD-4121/5ZLA; initial binding state, EMD-4122/5ZLB; codon reading state, EMD-4123/5ZLC; GTPase-activated state, EMD-4124/5ZLD; classical state, EMD-4125/5ZLE; hybrid state, EMD-4126/5ZLF. Cryo-EM micrographs and particle images have been deposited in the EMPIAR database (https://www.ebi. ac.uk/pdbe/emdb/empiar/) with accession code EMPIAR-10077. 
47. Rodnina, M. V. et al. Thiostrepton inhibits the turnover but not the GTPase of elongation factor G on the ribosome. Proc. Natl Acad. Sci. USA 96, 9586-9590 (1999).

48. Milon, P. et al. Transient kinetics, fluorescence, and FRET in studies of initiation of translation in bacteria. Methods Enzymol. 430, 1-30 (2007).

49. Rodnina, M. V. \& Wintermeyer, W. GTP consumption of elongation factor Tu during translation of heteropolymeric mRNAs. Proc. Natl Acad. Sci. USA 92 1945-1949 (1995).

50. Thanbichler, M. \& Böck, A. Purification and characterization of hexahistidinetagged elongation factor SelB. Protein Expr. Purif. 31, 265-270 (2003).

51. Paleskava, A., Konevega, A. L. \& Rodnina, M. V. Thermodynamics of the GTP-GDP-operated conformational switch of selenocysteine-specific translation factor SelB. J. Biol. Chem. 287, 27906-27912 (2012)

52. Thanbichler, M. \& Böck, A. Selenoprotein biosynthesis: purification and assay of components involved in selenocysteine biosynthesis and insertion in Escherichia coli. Methods Enzymol. 347, 3-16 (2002).

53. Kothe, U., Paleskava, A., Konevega, A. L. \& Rodnina, M. V. Single-step purification of specific tRNAs by hydrophobic tagging. Anal. Biochem. 356 , 148-150 (2006)

54. Fischer, N. et al. Towards understanding selenocysteine incorporation into bacterial proteins. Biol. Chem. 388, 1061-1067 (2007).

55. Konevega, A. L. et al. Purine bases at position 37 of tRNA stabilize codonanticodon interaction in the ribosomal A site by stacking and Mg2+ -dependent interactions. RNA 10, 90-101 (2004)

56. Rodnina, M. V., Fricke, R. \& Wintermeyer, W. Transient conformational states of aminoacyl-tRNA during ribosome binding catalyzed by elongation factor Tu. Biochemistry 33, 12267-12275 (1994).

57. Gromadski, K. B. \& Rodnina, M. V. Kinetic determinants of high-fidelity tRNA discrimination on the ribosome. Mol. Cell 13, 191-200 (2004).

58. Fischer, N. et al. Structure of the E. coli ribosome-EF-Tu complex at $<3 \AA$ resolution by Cs-corrected cryo-EM. Nature 520, 567-570 (2015).

59. Valle, M. et al. Cryo-EM reveals an active role for aminoacyl-tRNA in the accommodation process. EMBO J. 21, 3557-3567 (2002)

60. Stark, H. et al. Ribosome interactions of aminoacyl-tRNA and elongation factor Tu in the codon-recognition complex. Nat. Struct. Biol. 9, 849-854 (2002).

61. Scheres, S. H. RELION: implementation of a Bayesian approach to cryo-EM structure determination. J. Struct. Biol. 180, 519-530 (2012).

62. Chen, S. et al. High-resolution noise substitution to measure overfitting and validate resolution in $3 \mathrm{D}$ structure determination by single particle electron cryomicroscopy. Ultramicroscopy 135, 24-35 (2013).

63. Kucukelbir, A., Sigworth, F. J. \& Tagare, H. D. Quantifying the local resolution of cryo-EM density maps. Nat. Methods 11, 63-65 (2014).

64. Pettersen, E. F. et al. UCSF Chimera--a visualization system for exploratory research and analysis. J. Comput. Chem. 25, 1605-1612 (2004).

65. Kleywegt, G. J. \& Jones, T. A. Software for handling macromolecular envelopes. Acta Crystallogr. D 55, 941-944 (1999).

66. Brunger, A. T. Version 1.2 of the Crystallography and NMR system. Nat. Protocols 2, 2728-2733 (2007)

67. Brünger, A. T. et al. Crystallography \& NMR system: A new software suite for macromolecular structure determination. Acta Crystallogr. D 54, 905-921 (1998).

68. Adams, P. D. et al. PHENIX: a comprehensive Python-based system for macromolecular structure solution. Acta Crystallogr. D 66, 213-221 (2010)

69. DiMaio, F. et al. Improved molecular replacement by density- and energyguided protein structure optimization. Nature 473, 540-543 (2011).

70. DiMaio, F., Tyka, M. D., Baker, M. L., Chiu, W. \& Baker, D. Refinement of protein structures into low-resolution density maps using rosetta. J. Mol. Biol. 392 , 181-190 (2009).
71. Söding, J., Biegert, A. \& Lupas, A. N. The HHpred interactive server for protein homology detection and structure prediction. Nucleic Acids Res. 33, W244-W248 (2005)

72. Selmer, M. \& Su, X. D. Crystal structure of an mRNA-binding fragment of Moorella thermoacetica elongation factor SelB. EMBO J. 21, 4145-4153 (2002).

73. Soler, N., Fourmy, D. \& Yoshizawa, S. Structural insight into a molecular switch in tandem winged-helix motifs from elongation factor SelB. J. Mol. Biol. 370, 728-741 (2007)

74. Emsley, P., Lohkamp, B., Scott, W. G. \& Cowtan, K. Features and development of Coot. Acta Crystallogr. D 66, 486-501 (2010).

75. Keating, K. S. \& Pyle, A. M. Semiautomated model building for RNA crystallography using a directed rotameric approach. Proc. Natl Acad. Sci. USA 107, 8177-8182 (2010)

76. Chou, F. C., Sripakdeevong, P., Dibrov, S. M., Hermann, T. \& Das, R. Correcting pervasive errors in RNA crystallography through enumerative structure prediction. Nat. Methods 10, 74-76 (2013).

77. Afonine, P. V., Headd, J. J., Terwilliger, T. C. \& Adams, P. D. New tool: phenix. real space refine. Computational Crystallography Newsletter 43-44 (2013).

78. Terwilliger, T. C. Maximum-likelihood density modification. Acta Crystallogr. D 56, 965-972 (2000)

79. Karplus, P. A. \& Diederichs, K. Linking crystallographic model and data quality. Science 336, 1030-1033 (2012)

80. Winn, M. D. et al. Overview of the CCP4 suite and current developments. Acta Crystallogr. D 67, 235-242 (2011).

81. Bock, L. V. et al. Energy barriers and driving forces in tRNA translocation through the ribosome. Nat. Struct. Mol. Biol. 20, 1390-1396 (2013).

82. Pronk, S. et al. GROMACS 4.5: a high-throughput and highly parallel open source molecular simulation toolkit. Bioinformatics 29, 845-854 (2013).

83. Lindorff-Larsen, K. et al. Improved side-chain torsion potentials for the Amber ff99SB protein force field. Proteins 78, 1950-1958 (2010)

84. Berendsen, H. J. C., Grigera, J. R. \& Straatsma, T. P. The missing term in effective pair potentials. J. Phys. Chem. 91, 6269-6271 (1987).

85. Joung, I. S. \& Cheatham, T. E., III. Determination of alkali and halide monovalent ion parameters for use in explicitly solvated biomolecular simulations. J. Phys. Chem. B 112, 9020-9041 (2008).

86. Aduri, R. et al. AMBER Force Field Parameters for the Naturally Occurring Modified Nucleosides in RNA. J. Chem. Theory Comput. 3, 1464-1475 (2007).

87. Wang, J., Wang, W., Kollman, P. A. \& Case, D. A. Automatic atom type and bond type perception in molecular mechanical calculations. J. Mol. Graph. Model. 25 247-260 (2006)

88. Amadei, A., Linssen, A. B. \& Berendsen, H. J. Essential dynamics of proteins. Proteins 17, 412-425 (1993)

89. Schrader, J. M. \& Uhlenbeck, O. C. Is the sequence-specific binding of aminoacyl-tRNAs by EF-Tu universal among bacteria? Nucleic Acids Res. 39, 9746-9758 (2011)

90. Haruna, K., Alkazemi, M. H., Liu, Y., Söll, D. \& Englert, M. Engineering the elongation factor Tu for efficient selenoprotein synthesis. Nucleic Acids Res. 42 9976-9983 (2014)

91. Itoh, Y. et al. Decameric SelA•tRNA(Sec) ring structure reveals mechanism of bacterial selenocysteine formation. Science 340, 75-78 (2013).

92. Li, W. et al. Recognition of aminoacyl-tRNA: a common molecular mechanism revealed by cryo-EM. EMBO J. 27, 3322-3331 (2008)

93. Takyar, S., Hickerson, R. P. \& Noller, H. F. mRNA helicase activity of the ribosome. Cell 120, 49-58 (2005).

94. Budkevich, T. V. et al. Regulation of the mammalian elongation cycle by subunit rolling: a eukaryotic-specific ribosome rearrangement. Cell 158, 121-131 (2014).

95. Frank, J. \& Agrawal, R. K. A ratchet-like inter-subunit reorganization of the ribosome during translocation. Nature 406, 318-322 (2000). 
a

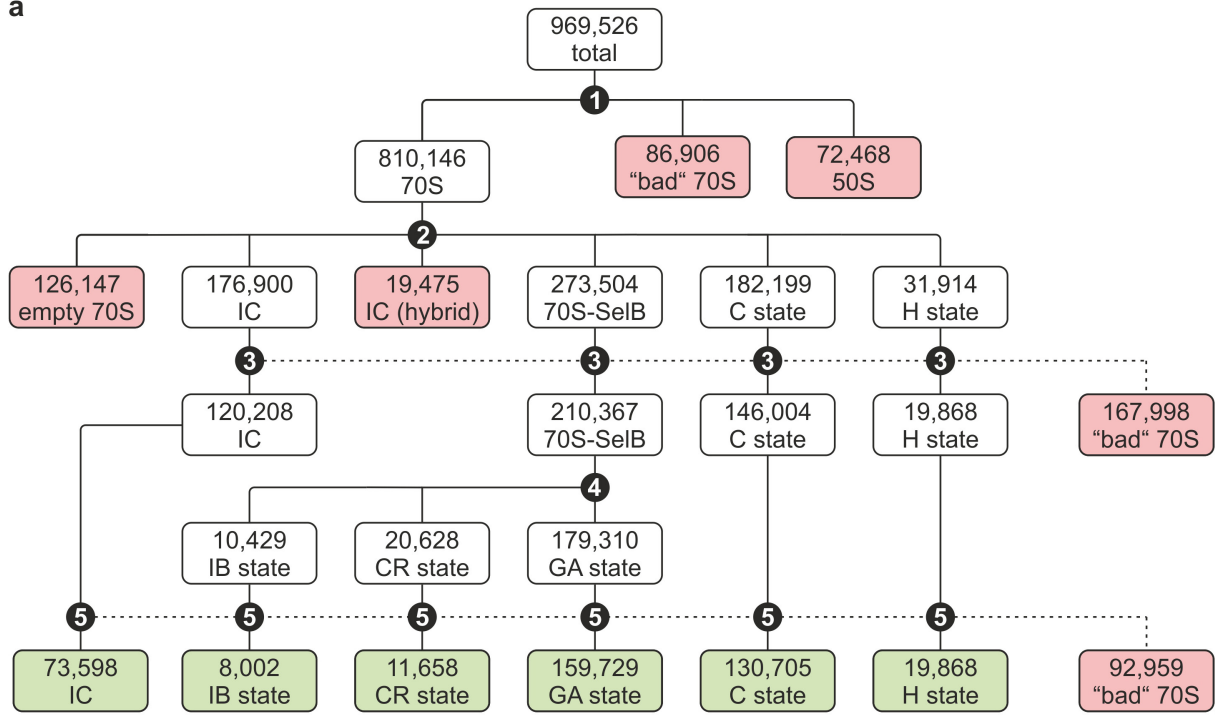

(1) SVC: $50 \mathrm{~S}$ subunits and low quality particles

2 SVC: Ligand occupancy

3 3D classification: Particle quality

4 Focused classification without alignment: TC conformation

$(5)$ SVC: Particle quality and ligand definition

Extended Data Figure 1 | Computational sorting of ribosome particle images. a, Hierarchical sorting scheme. Numbers refer to classification steps. SVC, supervised classification by projection matching; 'bad' 70S, the particles of low quality and/or those showing mixtures of different compositions and conformations; initial complex (hybrid) (IC (hybrid)), ratcheted state of the ribosome with one tRNA in the hybrid state. b, Mask

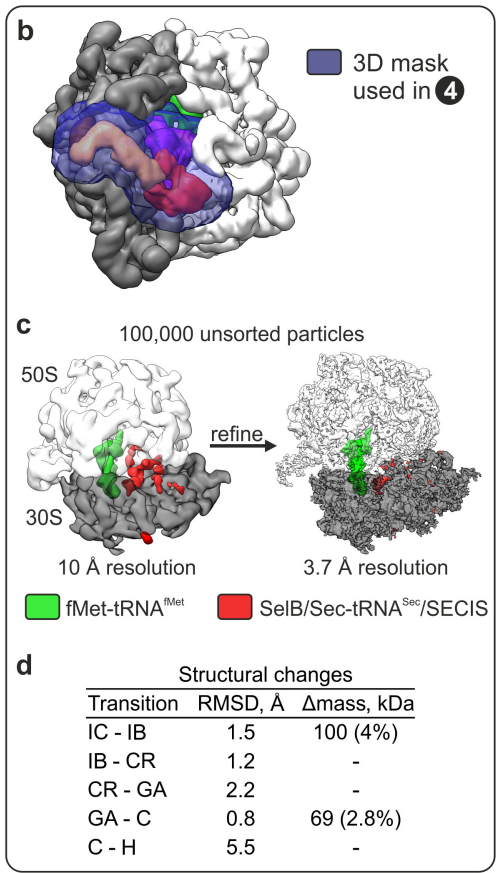

used for focused classification in step 4. c, Cryo-EM reconstruction from 100,000 random unsorted particle images. Left, initial refinement stage at $10 \AA$ A resolution showing only scattered density (red) for SelB, Sec-tRNA ${ }^{\mathrm{Sec}}$ and SECIS. Right, final cryo-EM map at $3.7 \AA$ resolution. d, Structural changes resolved by computational sorting as quantified by the r.m.s.d. and the changes in mass due to ligand binding and dissociation. 

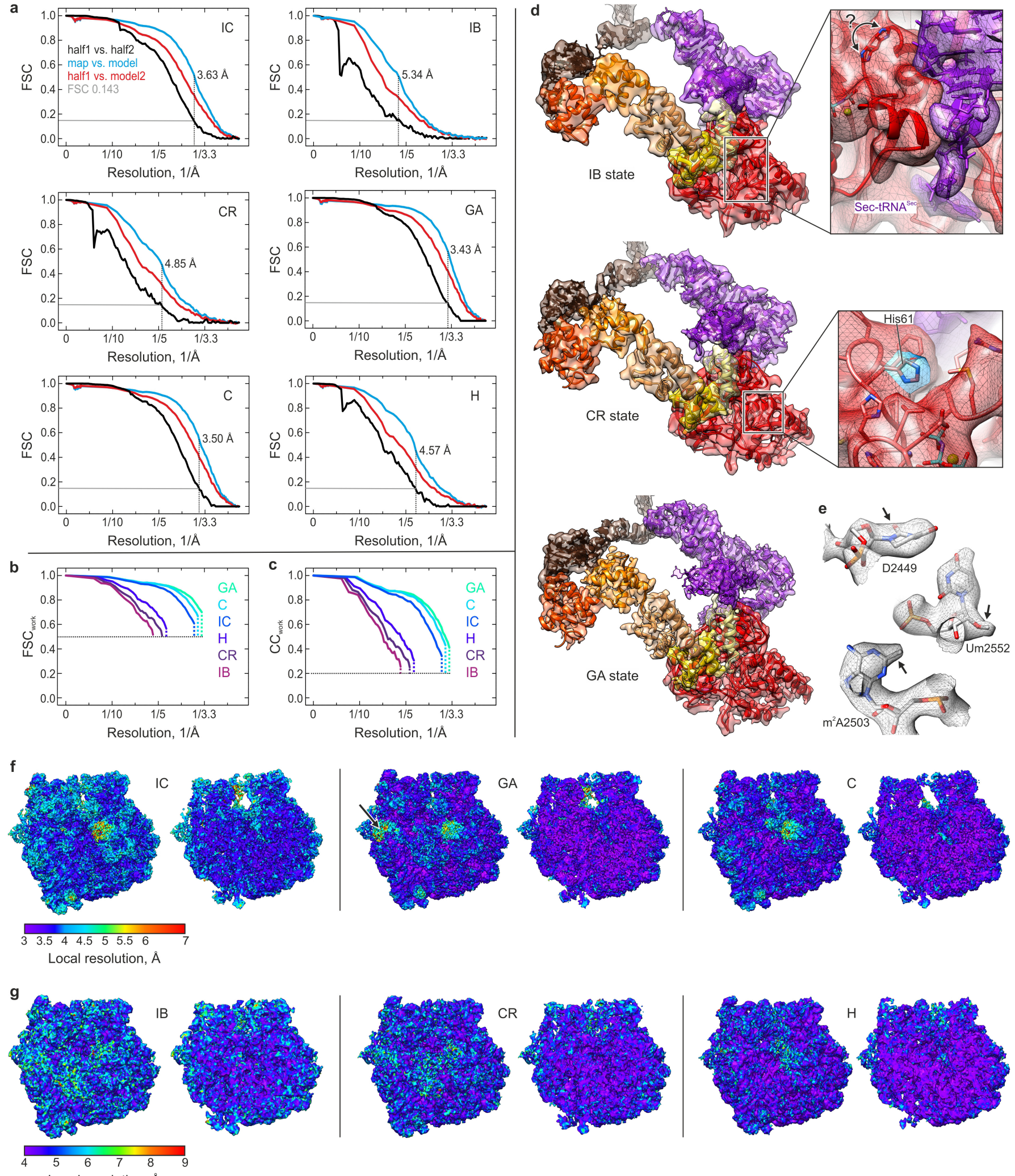

Extended Data Figure 2 See next page for caption. 
Extended Data Figure $2 \mid$ Cryo-EM map and model quality. a, Fourier shell correlation (FSC) curves and model validation. FSC curves are shown for each state. Black, the FSC curve computed between the masked independent half-maps from cryo-EM refinement (half1 and half2); blue, the FSC curve between the final cryo-EM map (map) and the final model (model); red, the FSC curve between half-map 1 (half1) and the model obtained by refinement only against half-map 2 (model2). The vertical black dashed line indicates the resolution according to the 0.143 criterion (grey line) and the maximum resolution at which the full atomic models were refined. b, FSC curves $\left(\mathrm{FSC}_{\text {work }}\right.$ ) computed for each state between reflections from solvent-flattened cryo-EM maps and model maps generated from refined atomic coordinates. Differences to $\mathbf{d}$ largely result from solvent-flattening (Methods). c, $\mathrm{CC}_{\text {work }}$ curves as obtained by refinement for each state. For reliable resolution estimates $\mathrm{CC}_{\mathrm{work}}$ is expected to be $>0.2$ in the highest resolution shell. d, Cryo-EM densities and models of the quarternary complex, SelB-GDPNP-Sec-tRNA ${ }^{\text {Sec }}$

SECIS-mRNA for each SelB-bound ribosome state. Densities are coloured as in Fig. $1 \mathrm{~b}$ and rendered at $\sim 2 \sigma$, except wh3, wh 4 and SECIS of the GTPase-activated state, which are rendered at $\sim 1.5 \sigma$. Top right: SectRNA $^{\text {Sec }}$ density (purple) in the initial binding state at $5.3 \AA$ resolution; the conformation of the invariant histidine 61 is not discernible (question mark). Middle right: Density for histidine 61 (blue) in the codon reading state indicating a partially flipped-in conformation. e, Densities for rRNA modifications as seen in the GTPase-activated state at 3.4 $\AA$ resolution. Densities are rendered at $\sim 3 \sigma$. Arrows denote the characteristic distortion of the nucleobase of D2449 and methyl groups for the other modified nucleotides. $\mathbf{f}, \mathbf{g}$, Cryo-EM reconstructions of the individual states (as indicated) coloured according to local resolution. Left: Surface view; right: Cut-away view. Heat maps are adjusted to the respective resolutions ranges of the cryo-EM maps in $\mathbf{f}$ and $\mathbf{g}$. The arrow denotes the substantially lower local resolution of SelB residues 485 to 614 in the GTPase-activated state; atomic models for such regions were correspondingly refined at lower resolution (Methods) 
a
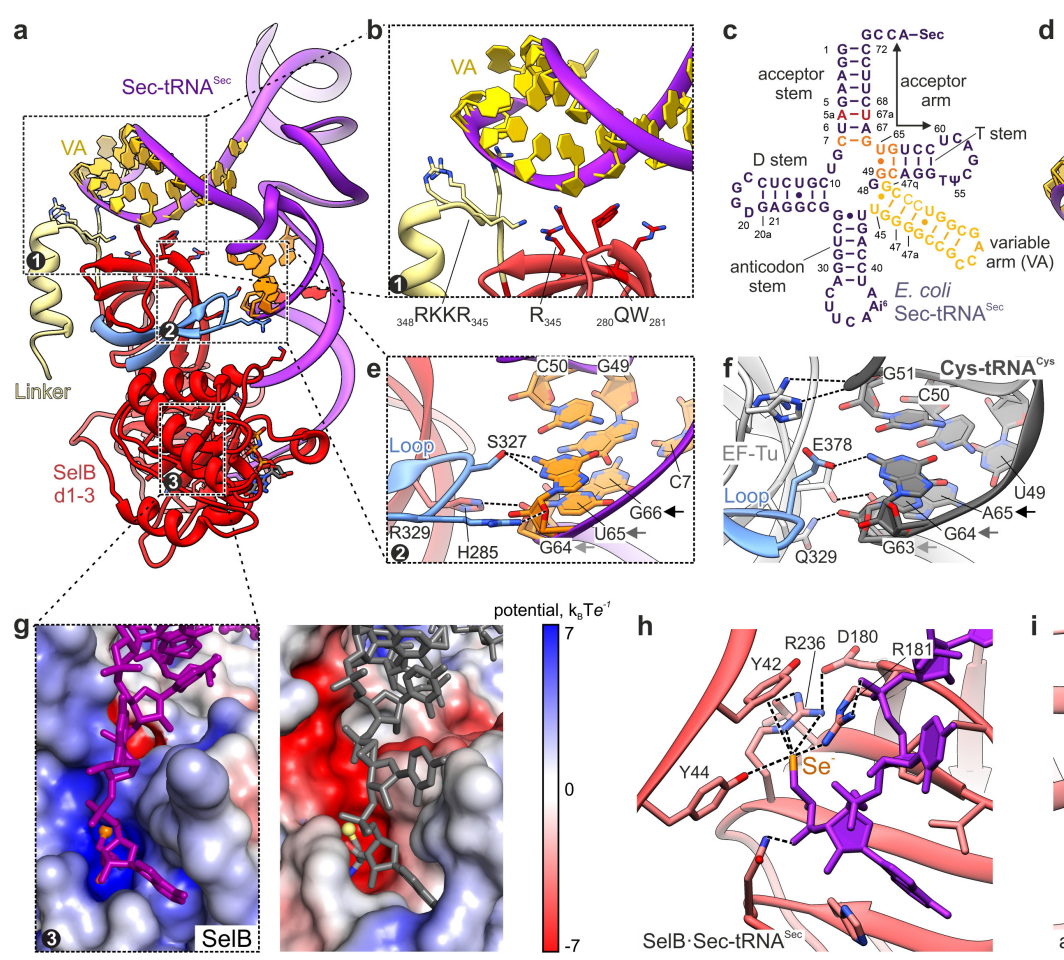
potential, $\mathrm{k}_{\mathrm{B}} T e^{-1}$

k

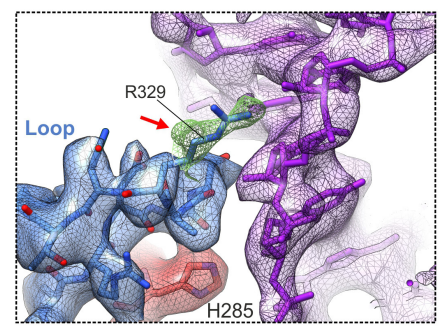

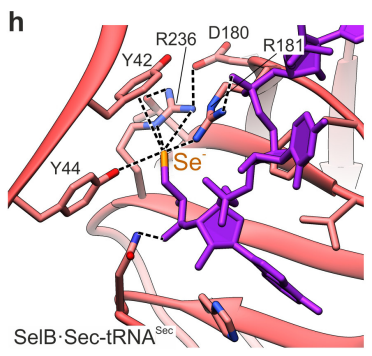

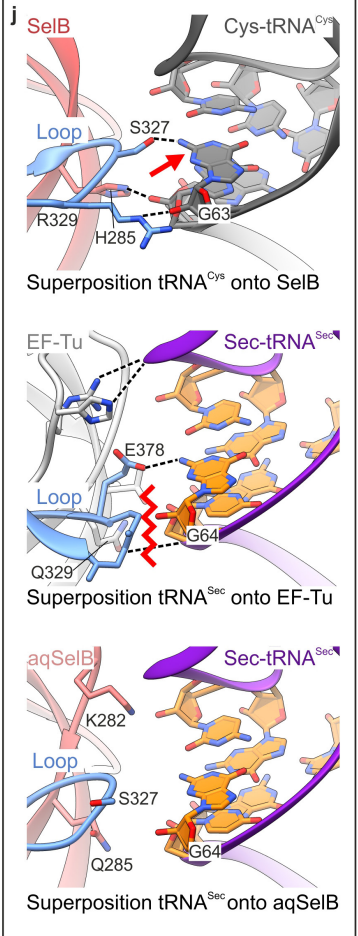

aqSelB.Cys

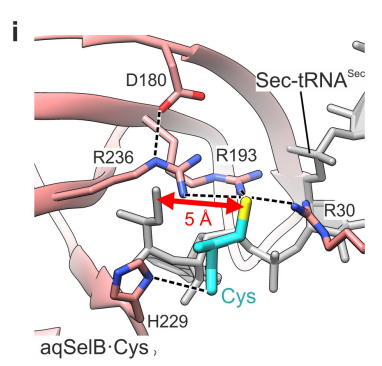

Superposition tRNA ${ }^{\mathrm{Sec}}$ onto aqSelB

Extended Data Figure 3 | See next page for caption.

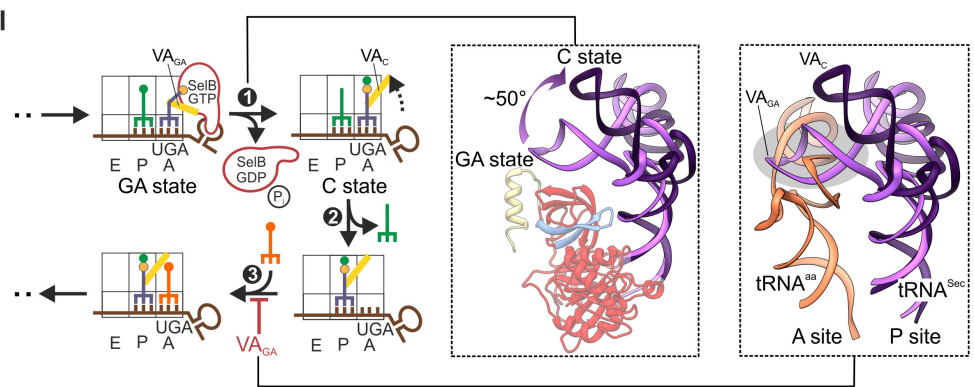


Extended Data Figure 3 Structural basis for the specificity of tRNA ${ }^{\mathrm{Sec}}$ recognition by SelB. All superpositions are based on domains 1 and 2 of SelB and EF-Tu, respectively. a, Overview of SelB-GDPNP-Sec-tRNA ${ }^{\text {Sec }}$ bound to the ribosome in the GTPase-activated state. Numbers refer to the close-ups in $\mathbf{b}$, e and $\mathbf{g}$, respectively, which depict specific SelB-

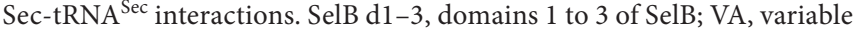
arm. b. Interactions between SelB (domain 3, red, and the linker, residues $340-363$ in khaki) and the backbone of the extra-long variable arm of tRNA $^{\text {Sec. }}$. These interactions do not form in the canonical EF-Tu-aa-tRNA complexes. $c$, The structure of tRNA ${ }^{\text {Sec }}$. In contrast to canonical tRNAs, tRNA $^{\text {Sec }}$ entails an additional base-pair in the acceptor stem (A5a-U67a, red), a unique sequence in the T-stem, the so-called antideterminant box (C7-G66, G49-U65 and C50-G64, orange), and a very long variable arm (yellow). d, Superposition of tRNA ${ }^{\text {Sec }}$ (purple) and the canonical tRNA ${ }^{\text {Cys }}$ (dark grey) in the complex with SelB-GDPNP and EF-Tu-GDPNP, respectively. The structure of the canonical EF-Tu-GDPNP-Cys-tRNA ${ }^{\text {Cys }}$ is from PDB structure 1B23 (ref. 11). Note the distortion in the acceptor stem of tRNA ${ }^{\text {Sec }}$ (residues G1-U6/G72-A67) as compared to tRNA ${ }^{\text {Cys }}$ that compensates for the additional base pair. As a result of the distortion, the main factor-binding sites of both tRNAs, the CCA end and the T-stem, overlap and the bases are arranged in register ('in'). In the T-stem, the bases are shifted by exactly one base pair (' $\Delta 1$ ') between the two tRNAs, which shifts the bases out of register ('out') in the acceptor stems of the two tRNAs. e, Specific interaction between the extended loop (blue) in SelB domain 3 and the antideterminant box in the T-stem of tRNA ${ }^{\text {Sec }}$. f, Sequence-specific interaction between the loop (blue) in EF-Tu domain 3 and the T-stem of tRNA ${ }^{\text {Cys }}$ (PDB ID 1B23 ref. 11). Note the different structure of the hairpin-loop as compared to SelB, contributing to the particularly low affinity of EF-Tu for tRNA ${ }^{\text {Sec }}$ (ref. 89) (see also k, middle panel). g, Charge distribution in the amino-acid-binding pockets of SelB (left) and EF-Tu (right). The colour bar on the right denotes the electrostatic potential in $k_{\mathrm{B}} T \mathrm{e}^{-1}$. Note the similar geometry of tRNA binding in the two complexes. In SelB, the pocket is positively charged, facilitating the interaction with the negatively charged selenol group of Sec-tRNA ${ }^{\mathrm{Sec}}$ and the discrimination against the precursors of Sec-tRNA ${ }^{\mathrm{Sec}}$ biosynthesis, deacylated tRNA ${ }^{\text {Sec }}$ and Ser-tRNA ${ }^{\text {Sec }}$, as well as against most canonical tRNAs. In EF-Tu, the amino-acid-binding pocket is negatively charged, thereby discriminating against Sec-tRNA ${ }^{\mathrm{Sec}}$. $\mathbf{h}$, Recognition of the selenol group in Sec-tRNA ${ }^{\text {Sec }}$ by SelB. The positively charged, highly conserved Arg181 and Arg236 in SelB contact the negatively charged selenol group $\left(\mathrm{Se}^{-}\right.$, orange), whereas the aromatic ring of Tyr42 stacks onto the selenol group. The importance of these residues was demonstrated by mutational analysis ${ }^{9,90}$. The universally conserved Asp180, which is also important for Sec-tRNA ${ }^{\text {Sec }}$ binding $^{9}$, forms a secondary binding shell stabilizing Arg236. i, Solvent-exposed amino-acidbinding site as found in the crystal structure of isolated Aquifex aeolicus (aq) SelB-GDPNP co-crystallized with L-cysteine as Sec mimic (PDB ID 4ZU9 $)^{8}$. The position of Cys deviates by $5 \AA$ from the amino acid position in the present SelB-GDPNP-Sec-tRNA ${ }^{\text {Sec }}$ structure (grey), resulting in a distinct interaction pattern and a more solvent-exposed binding site of the Cys. j, Superpositions of SelB-GDPNP-Sec-tRNA ${ }^{\text {Sec }}$ and EF-Tu-GDPNPCys-tRNA ${ }^{\text {Cys }}$ revealing differences in T-stem recognition by the loop in SelB domain 3 versus EF-Tu. Top: The loop structure in SelB is compatible with binding of canonical tRNA ${ }^{\text {Cys }}$. Other tRNAs with different $\mathrm{T}$ stem sequences (particularly at position 63, red arrow) may be unfavourable for the interaction with the conserved Ser327 in SelB. Middle: The loop structure in EF-Tu is incompatible with the conformation of the antideterminant box (orange) in tRNA ${ }^{\text {Sec }}$. Bottom: In isolated aqSelB (PDB ID 4 ZU9 $)^{8}$, the loop adopts a similar conformation as in the SelB ternary complex, suggesting a conserved loop structure that facilitates recognition of tRNA ${ }^{\text {Sec }} . \mathbf{k}$, Experimental density depicting the interaction of SelB with the acceptor and T stem region of tRNA ${ }^{\mathrm{Sec}}$ in the GTPase-activated state (rendered at $2 \sigma$ ) suggesting alternative conformations of $\operatorname{Arg} 329$; green mesh, density rendered at $1 \sigma . \mathbf{1}$, Scheme depicting the functional importance of rearrangements of the tRNA ${ }^{\text {Sec }}$ variable arm for translation elongation after Sec incorporation. (1) Upon release from SelB, tRNA ${ }^{\text {Sec }}$ accommodates in the aminoacyl site in the classical state. The variable arm undergoes a large reorientation from a SelB-bound distorted conformation in the GTPase-activated state to its free ground state conformation in the classical state (left close-up); the latter ground state conformation is similar to the conformation of the variable arm found in complexes of tRNA ${ }^{\text {Sec }}$ with its conversion enzymes, seryl-tRNA synthetase ${ }^{6}$ and SelA ${ }^{91}$. (2) After peptide bond formation (resulting in the hybrid state, not shown), peptidyl-tRNA ${ }^{\mathrm{Sec}}$ is translocated to the classical peptidyl site. (3) Binding of the next aa-tRNA (orange) is only compatible with the variable arm adopting the classical state conformation; the GTPase-activated stateconformation of the variable arm would sterically interfere with binding of the next aa-tRNA (right close-up). The long variable arm of canonical class 2 aa-tRNA is not distorted upon EF-Tu binding ${ }^{92}$ and, consequently, does not require any changes during translation elongation. 


\section{RESEARCH ARTICLE}

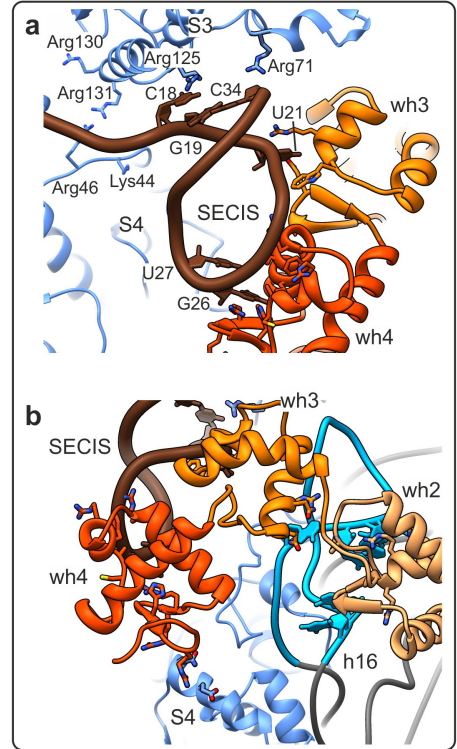

C

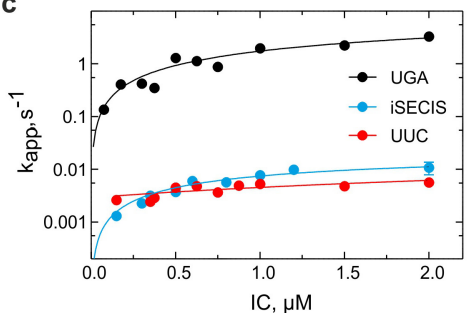

d

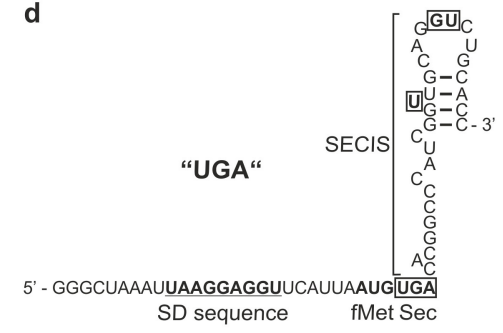

f

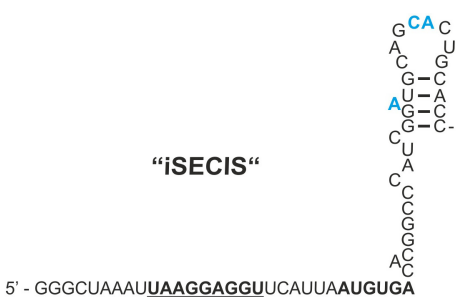

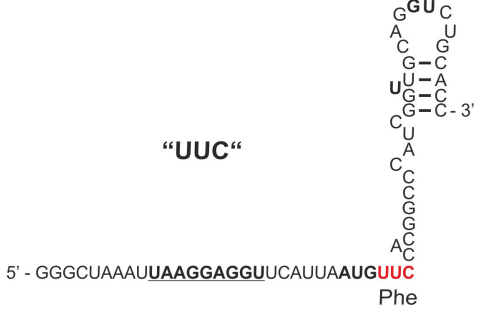

Extended Data Figure 4 | The SelB-SECIS interactions on the ribosome and kinetic analysis of GTP hydrolysis. a, Close-up of the SECISribosome interactions in the GTPase-activated state. Ribosomal proteins S3, S4, and S5 contribute to the helicase activity of the ribosome ${ }^{93}$. Arg130 and Arg131 of S3 and Lys44 and Arg46 of S4 are important for mRNA unwinding ${ }^{93}$. Other potential helicase elements are Arg71 and Arg125 in S3, which interact with the base of the SECIS hairpin and may thereby facilitate unwinding of the mRNA secondary structure elements. The mode of SECIS recognition by SelB on the ribosome is similar to that in isolated SelB domain 4-SECIS complexes, including three essential bulged nucleotides in the SECIS (U21, G26 and U27; present data and refs 9, 25 and 72). b, Interaction of SelB domain 4 with the shoulder of the 30 S subunit. Winged-helix motif 4 (wh4) of SelB forms salt bridges with protein S4, while wh 2 and wh3 embrace helix 16 of 16 S rRNA by interacting mainly with the rRNA backbone. c, Concentration dependence of the apparent rate of GTP hydrolysis by SelB measured with increasing concentrations of ribosomes programmed with mRNAs 'UGA' (black), 'iSECIS' (blue) and 'UUC' (red). d, mRNA construct 'UGA' used for structural and kinetic analyses containing the cognate UGA codon coding for Sec and the functional minimal $f d h F$-SECIS. The Sec codon and the essential bases of the SECIS are indicated by boxes; SD, Shine-Dalgarno sequence. e, f, mRNA constructs 'iSECIS' and 'UUC' used for kinetic analysis. Changes in comparison to the 'UGA' mRNA are indicated in blue (iSECIS) and red (UUC). 
a IC

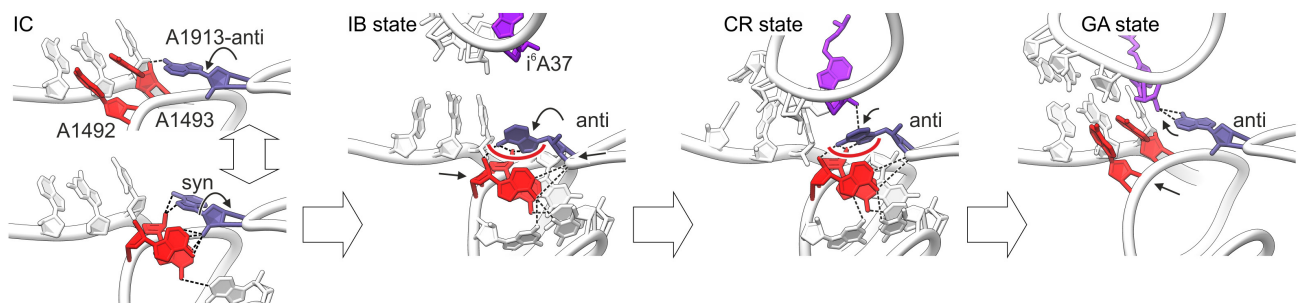

b
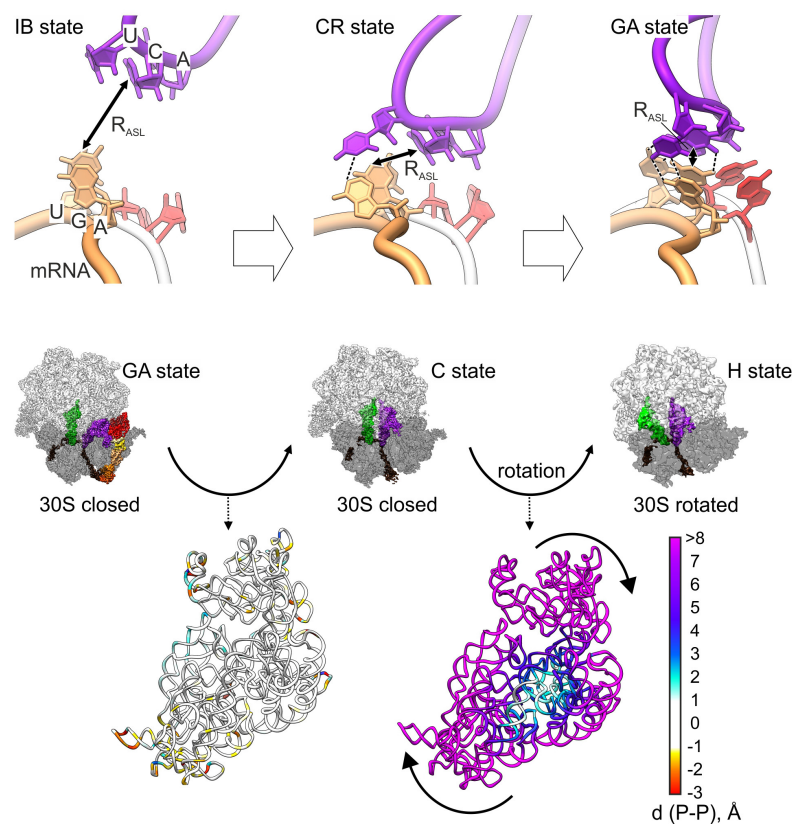
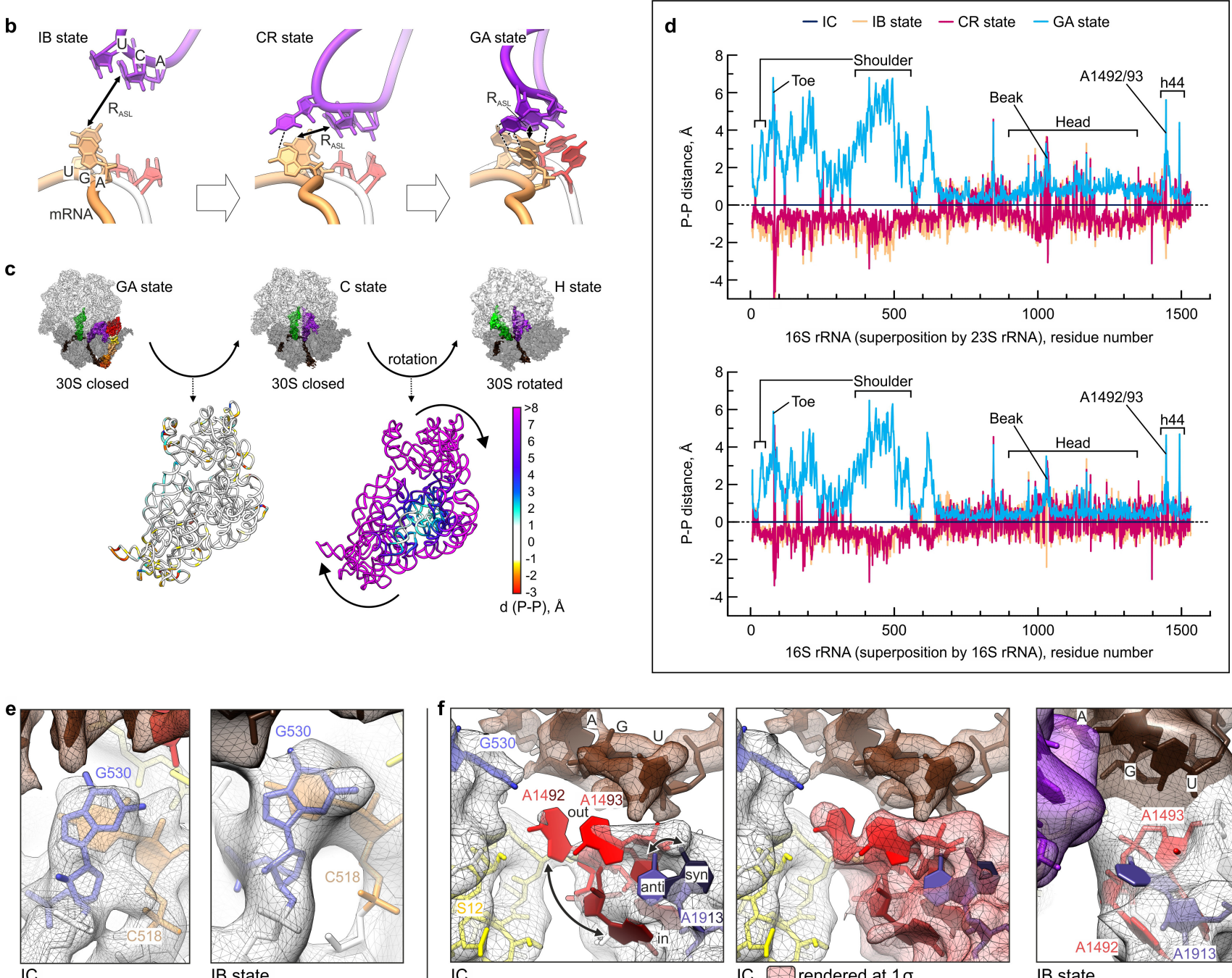

IB state

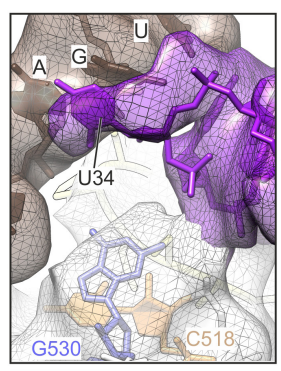

CR state

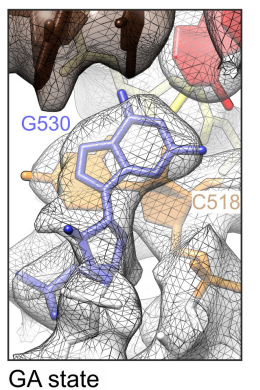

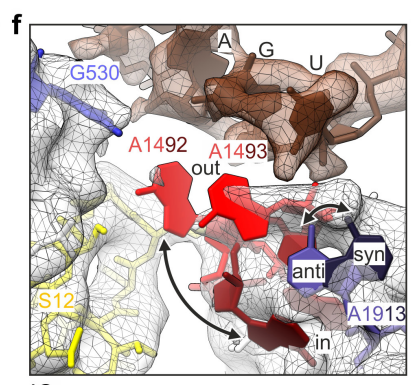

IC

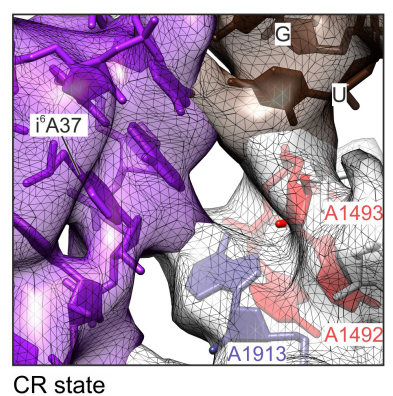

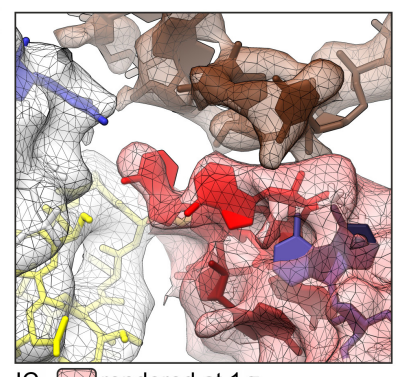

IC rendered at 10

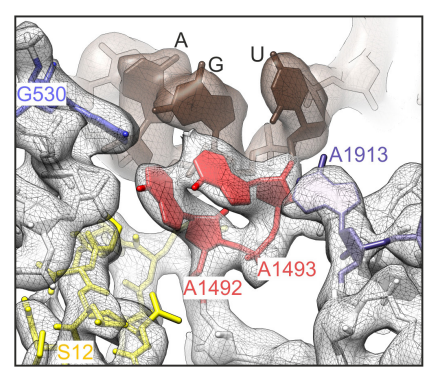

GA state

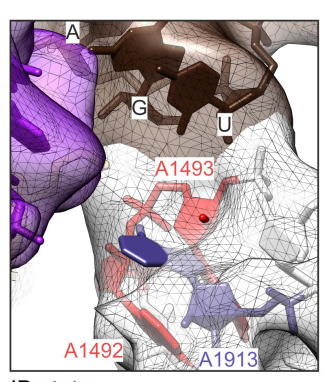

IB state

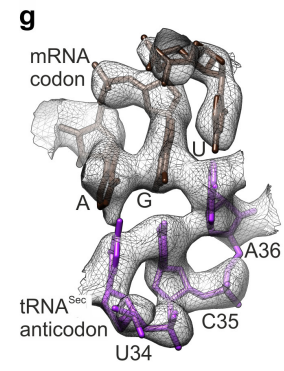

GA state

Extended Data Figure 5 | See next page for caption. 
Extended Data Figure 5 | Local and global conformational changes of the 30S subunit. a, Rearrangements of A1913 in H69 of 23S rRNA. A1913 stabilizes A1492 and A1493 and guides Sec-tRNA ${ }^{\text {Sec }}$ to the mRNA codon by interaction with isopentenyl-A37 (i6A37) of tRNA ${ }^{\mathrm{Sec}}$. The respective conformation (syn or anti) is denoted; dashed lines indicate potential interactions with distances $\leq 4 \AA$. $\mathbf{b}$, Close-up of the distance $\mathrm{R}_{\mathrm{ASL}}$ between the UGA (N1 of G) and anticodon of tRNA ${ }^{\text {Sec }}(\mathrm{N} 3$ of C35). Note the potential interaction between the third codon position and the tRNA anticodon in the codon reading state. c, Global conformational changes of the $30 \mathrm{~S}$ subunit upon accommodation (GTPase-activated to classical) and hybrid state formation (classical to hybrid). tRNA accommodation in the aminoacyl site does not cause major 30S subunit rearrangements, in contrast to the eukaryotic system, where tRNA accommodation correlates with a large-scale conformational change ${ }^{94}$. Subsequent tRNA hybrid state formation is coupled to the well-known rotational movement of the 30 Subunit $^{95}$. The typical rotational changes all over the $30 \mathrm{~S}$ subunit are clearly distinct from the changes observed upon domain opening and closure, which are mainly found at the shoulder region (Fig. 3a). The heat map quantifies the movements of $16 \mathrm{~S}$ rRNA backbone phosphate atoms as obtained by superposition on $23 \mathrm{~S}$ rRNA of the initial complex state (Fig. 3a). d, Deviations of the 16S rRNA backbone phosphates in the initial binding, codon reading, or GTPase-activated states from the initial complex superimposed on 23S rRNA (top) or 16S rRNA (bottom).
Negative values correspond to the $30 \mathrm{~S}$ domain opening, positive values to domain closure. Landmarks of the $30 \mathrm{~S}$ subunit are indicated.

e, f, Experimental densities of the decoding centre region rendered at $2-2.5 \sigma$, if not indicated otherwise. For better visibility, densities for tRNA $^{\mathrm{Sec}}$ were omitted for the GTPase-activated state. e, Anti-conformation of G530 and stacking interaction with C518 of 16S rRNA as seen in the different states. Bottom left: Note the density of tRNA ${ }^{\text {Sec }}$ (purple) suggesting a partial interaction of U34 with the mRNA codon in the codon reading state. f, Different conformational states of A1492, A1493 (red) of $16 \mathrm{~S}$ rRNA and A1913 (slate blue) of $23 \mathrm{~S}$ rRNA. Top left: In the initial complex the reduced densities for the three nucleotides indicate a dynamic equilibrium between two states: (1) A1492, A1493 flipped out ('out', red) with A1913 in anti-configuration ('anti', slate blue) and (2) A1492, A1493 flipped in ('in', dark red) with A1913 in syn-configuration ('syn', dark slate blue). Top middle: The dynamic nucleotides in the initial complex are discernible at lower threshold (red mesh, density at $\sim 1 \sigma$ ). Top right and bottom left: Density in the initial binding and codon reading states is compatible with the bases of A1492 and A1493 flipped-in and A1913 in the anti-configuration. Bottom right: In the GTPase-activated state, A1492 and A1493 are flipped-out and A1913 remains in the anti-conformation. g, Density for the codon-anticodon interaction in the GTPase-activated state rendered at $\sim 3 \sigma$. 


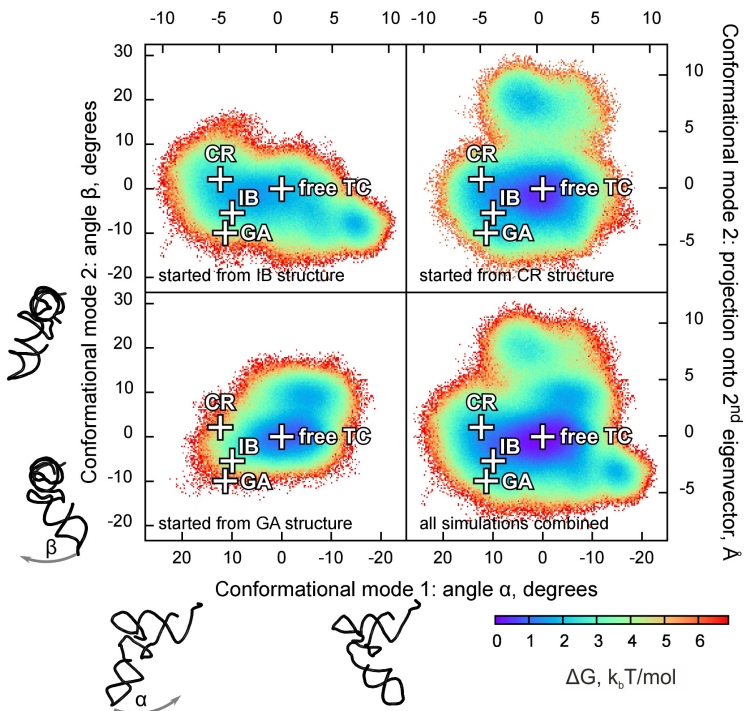

Extended Data Figure 6 | Molecular dynamics simulations of the free ternary complex. a, tRNA ${ }^{\mathrm{Sec}}$ dynamics obtained by molecular dynamics simulations of the free SelB-GTP-Sec-tRNA ${ }^{\text {Sec }}$ ternary complex. The free energy landscape for the conformational dynamics of the tRNA ${ }^{\mathrm{Sec}}$ body, excluding the variable arm, is shown as a heat map. The free energy was estimated from separate simulations starting from the structures of the ternary complex bound to the ribosome in initial binding, codon reading, and GTPase-activated states (upper and lower left panels), as well as from all simulations combined (lower right panel). The results of different simulations have a large overlap, especially in regions of low free energy. The crosses denote the tRNA conformations in the ribosomebound cryo-EM states and the free energy minimum of the free ternary complex found in the molecular dynamics simulations. b. Weak coupling between the conformational dynamics of tRNA ${ }^{\mathrm{Sec}}$ excluding the variable b
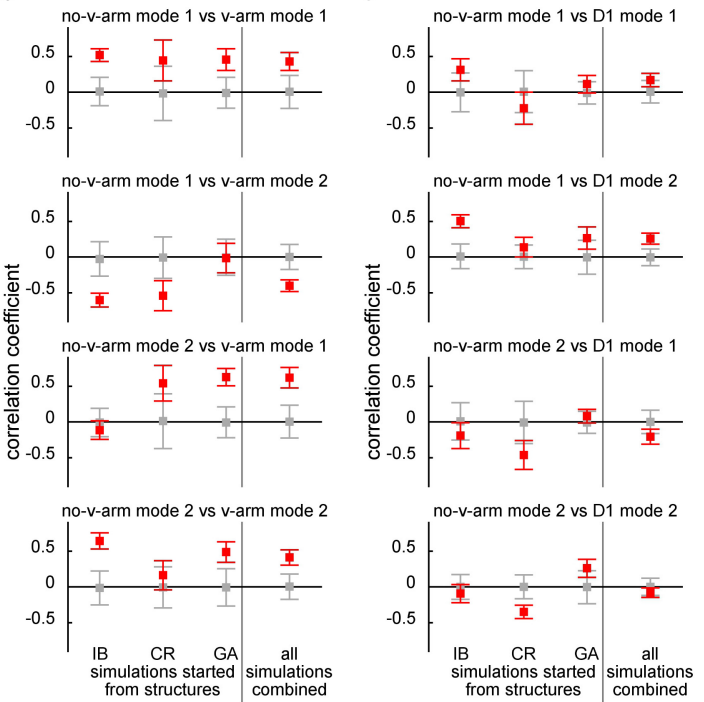

arm (no-v-arm) and of the variable arm (v-arm). Each panel shows the correlation coefficients for a pair of conformational modes (red points, with standard deviation) for the simulations starting from each state (initial binding, codon reading, and GTPase-activated), as well as for all simulations combined. The grey bars denote the standard deviation of the correlation coefficient expected from random drawing of projections. All pairs of modes show a small but significant correlation, indicating a weak coupling. c, Coupling between tRNA dynamics (no-v-arm modes) and SelB motions (SelB domain 1, D1, relative to domain 2, D2). Only a weak overall correlation is seen between no-v-arm mode 1 and D1 mode 2 . The three ribosome-bound conformations do not change much with regards to no-v-arm mode 1 (Fig. 3b), which suggests that there is no direct coupling between tRNA dynamics and SelB motions on the ribosome. 


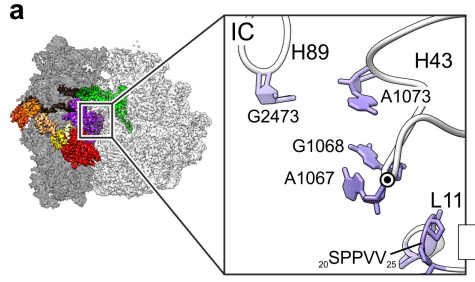

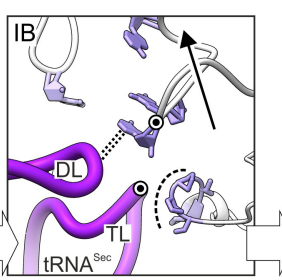

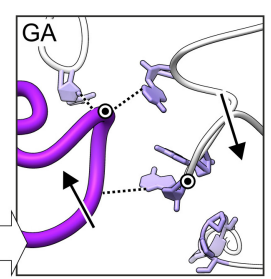

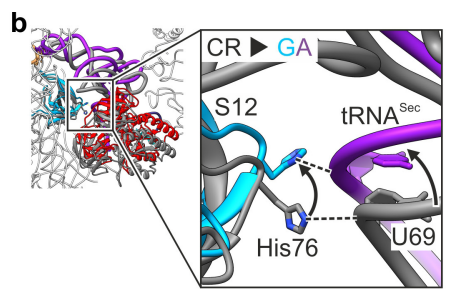

C

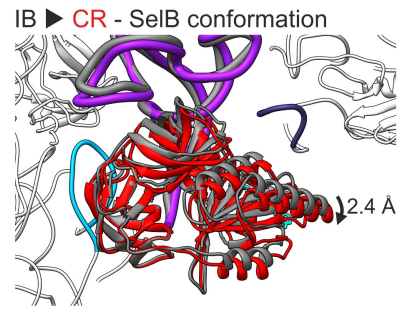

d $\mathrm{CR}>\mathrm{GA}$ - SelB conformation

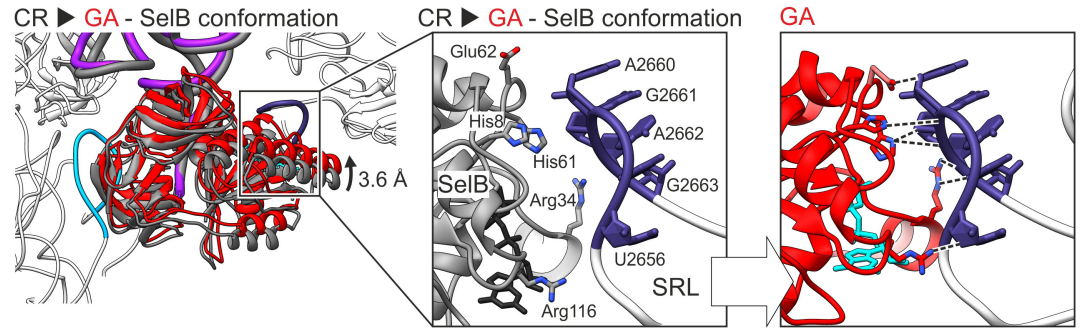

e

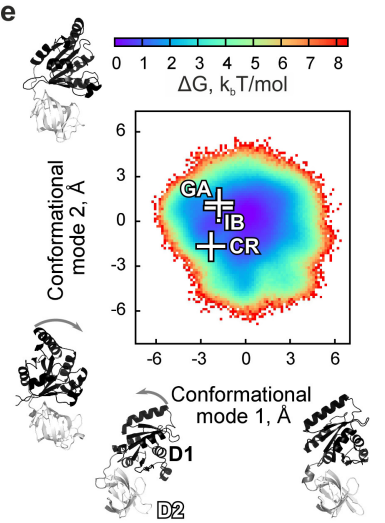

f

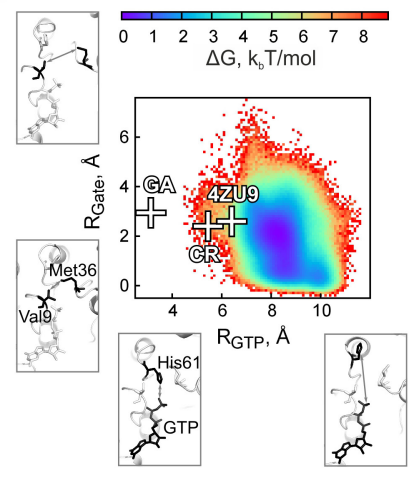

g

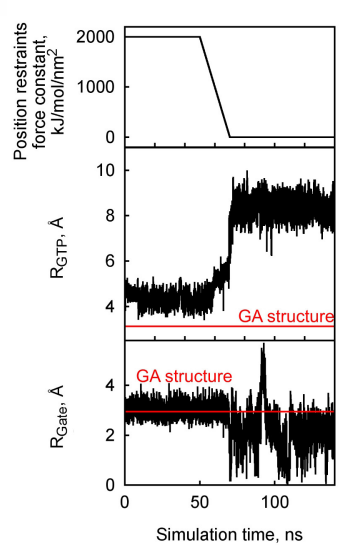

h

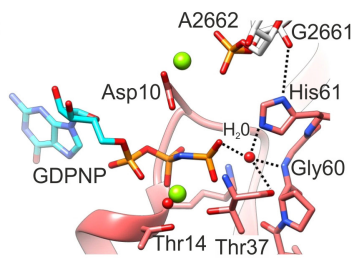

70S.SelB.GDPNP

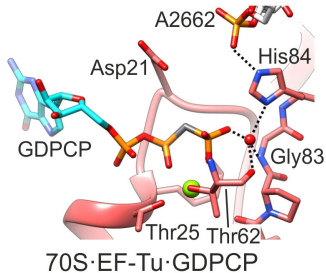

Extended Data Figure 7 | Role of L11-rRNA arm, protein S12 and the dynamics of SelB domains. a, Role of L11-rRNA arm in guiding SectRNA $^{\text {Sec }}$ towards H89. Residues of protein L11, H43 and H89 of $23 \mathrm{~S}$ rRNA are shown in mauve; dashed lines mark interactions with the D loop (DL) and T loop (TL) of tRNA ${ }^{\mathrm{Sec}}$ (purple); arrows denote changes with respect to the preceding state. $\mathbf{b}$, Protein S12 as a pivot for tRNA ${ }^{\text {Sec }}$ movement. S12 His7 6 interacts with the backbone of tRNA ${ }^{\text {Sec }}$ (residue U69) guiding the tRNA from its position in the codon reading state (grey) to the GTPaseactivated state (coloured). c, Inter-subunit rearrangement in SelB upon transition from initial binding to codon reading. Shown is the small movement observed in addition to the rotation of SelB relative to the $30 \mathrm{~S}$ shoulder as depicted in Fig. 4f, upper panel. d, SelB residues facilitating SRL docking. Left and middle: Spontaneous fluctuations within SelB that are required-in addition to the rotation upon $30 \mathrm{~S}$ domain closure (shown in Fig. 4, lower panel) - for transition from codon reading (grey) to the GTPase-activated state (red). Middle: 30 S domain closure and tRNA ${ }^{\mathrm{Sec}}$ repositioning re-align SelB and in particular its long polar residues towards the SRL. Right: SelB-SRL interactions resulting from docking in the GTPase-activated state. e, Motions of SelB domain 1 (D1) relative to domain 2 (D2) in the molecular dynamics simulations of the free

ternary complex. SelB domain 1 can rapidly and spontaneously sample arrangements required for the transitions from initial binding to codon reading and GTPase-activated states. $f$, Minimum distance between the imidazole ring of SelB His61 and $\gamma$-phosphate/oxygens of GTP $\left(\mathrm{R}_{\mathrm{GTP}}\right)$ and the minimum distance between the Van der Waals radii of Val9 and Met36 $\left(\mathrm{R}_{\mathrm{Gate}}\right)$, residues adjacent to His61 in SelB and analogous to the presumed 'gate' in EF-Tu ${ }^{3}$. In the free ternary complex, the distance between GTP and His61 of SelB is larger than in the GTPase-activated state on the ribosome, although Val9 and Met36 can freely move apart. Distances for the crystal structure of SelB-GDPNP are labelled 4ZU9 (ref. 8). g, Metastable SelB conformation in the GTPase-activated state. SelB in the GTPase-activated state was taken as initial structure for molecular dynamics simulations. During the first $50 \mathrm{~ns}$ of the simulations, the positions of heavy atoms are restrained to allow equilibration of the solvent (top panel). As soon as the restraints are released (50-70 ns), the distance between His61 and GTP increases (middle panel), and residues Val9 and Met36 resume fluctuations between open and closed conformations. h, Comparison of the active sites in SelB and EF-Tu. Top: GTPase centre of SelB in the GTPase-activated state. Bottom: Activated GTPase centre of EF-Tu on the ribosome (PDB ID 4V5L) ${ }^{35}$. 
Extended Data Table 1 | Data collection and model refinement

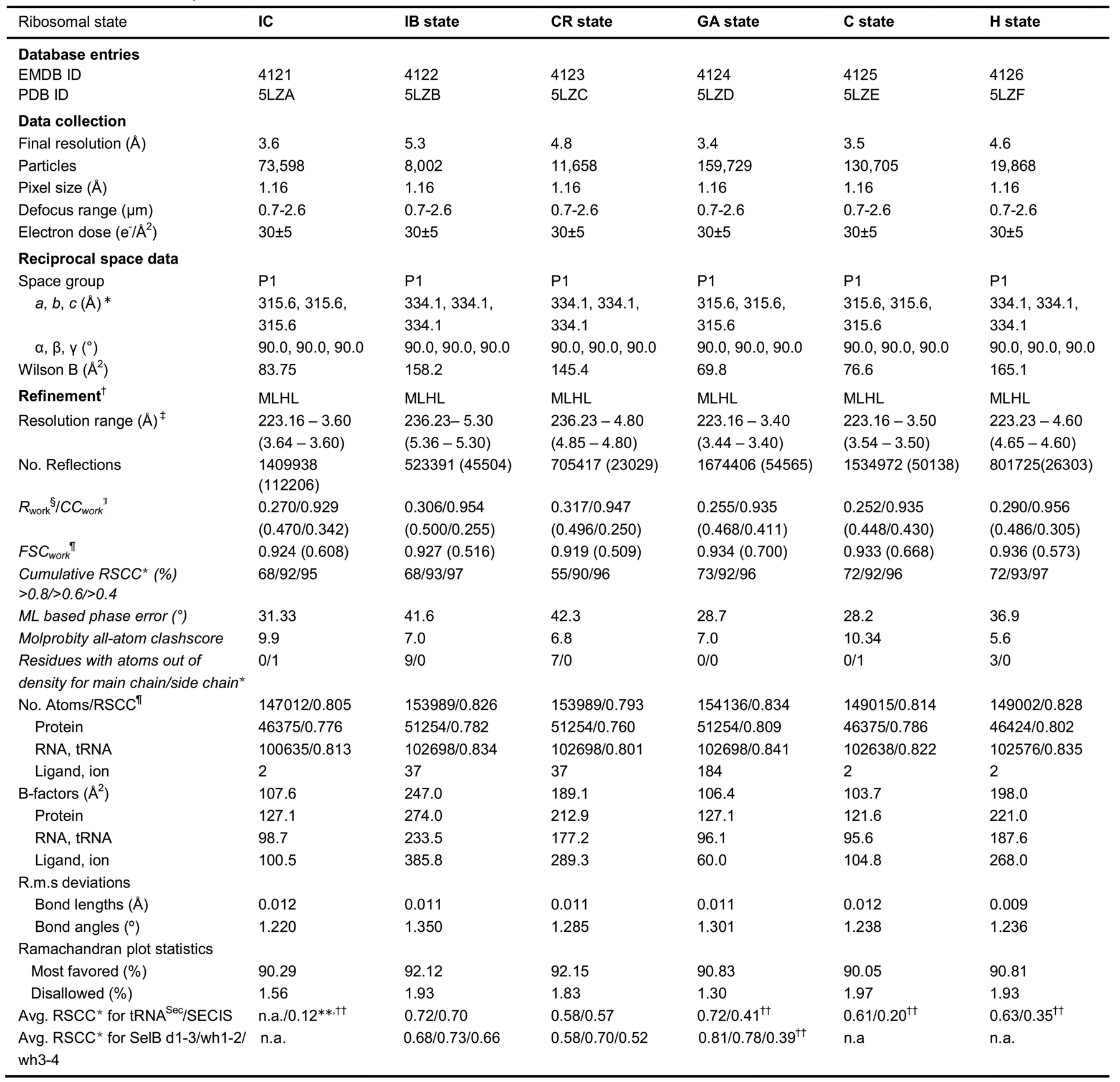

*For refinement, maps at $\geq 4.6 \AA \AA$ resolution were cropped to $280 \times 280 \times 280$ pixels; maps at $\leq 3.6 \AA$ resolution were resampled to $400 \times 400 \times 400$ pixels, corresponding to a pixel size of $0.789 \AA$.

tRefinement target: MLHL maximum likelihood with experimental phase probability distribution.

$\ddagger$ Highest resolution shell is shown in parenthesis.

$\S R_{\text {work: }}: \Sigma|| \mathrm{F}_{\mathrm{EM}}|-| \mathrm{F}_{\mathrm{MODEL}}|| /\left|\mathrm{F}_{\mathrm{EM}}\right|$, where $\mathrm{F}_{\mathrm{EM}}$ are structure factors calculated on the basis of solvent flattened EM map and $\mathrm{F}_{\text {MODEL }}$ are structure factors calculated from the refined model. The structure factors belonged to the working set which was used for reciprocal space refinement.

$\| \mathrm{CC}_{\text {work }}=$ Pearson correlation coefficient calculated between $\mathrm{F}_{\mathrm{EM}}$ and $\mathrm{F}_{\mathrm{MODEL}}$.

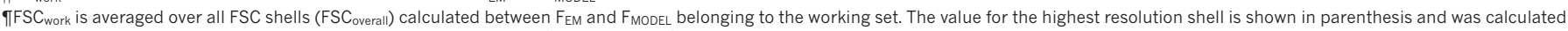
using the form: $\mathrm{FSC}_{\text {work(shell })}=\Sigma\left(\mathrm{F}_{\mathrm{EM}} \times \mathrm{F}_{\text {MODEL }} \cos (\Delta\right.$ phase $\left.)\right) /\left(\left(\mathrm{v}\left(\Sigma\left|\mathrm{F}_{\mathrm{EM}}\right|^{2}\right) \times\left(\mathrm{v}\left(\Sigma\left|\mathrm{F}_{\mathrm{MODEL}}\right|^{2}\right)\right)\right.\right.$.

\#Residue-averaged real space local correlation coefficient in region of model (RSCC) to EM map calculated with RESOLVE ${ }^{78}$.

*Calculated with RESOLVE ${ }^{78}$

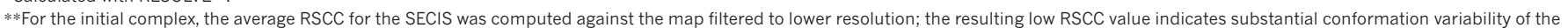
SECIS.

††Modelled based on cryo-EM map filtered to lower resolution. 